INRA Prod. Anim., 2017, 30 (1), 57-76

\section{Enjeux et outils du traitement raisonné contre les strongles gastro-intestinaux chez les bovins et les petits ruminants}

\author{
N. RAVINET ${ }^{\prime}$, C. CHARTIER ${ }^{1}$, H. HOSTE ${ }^{2}$, M. MAHIEU ${ }^{3}$, A. DUVAUCHELLE-WACHE ${ }^{4}$, \\ A. MERLIN, N. BAREILLE', P. JACQUIET', A. CHAUVIN ${ }^{1}$ \\ ${ }^{\prime}$ BioEpAR, INRA, Oniris, CS40706, 44307, Nantes, France
}

2 UMR INRA/ENVT 1225 IHAP, Ecole Nationale Vétérinaire de Toulouse, BP 87614, 31076, Toulouse, France ${ }^{3}$ INRA UR 143, Domaine de Duclos, 97170, Petit-Bourg, Guadeloupe ${ }_{4}^{4}$ Institut de l'Elevage, UMT Santé des troupeaux bovins, 44307, Nantes, France Courriel : christophe.chartier@oniris-nantes.fr

Chez les ruminants ayant accès au pâturage, le contrôle de l'infestation par les strongles gastrointestinaux repose essentiellement sur les traitements anthelminthiques, mais leur usage insuffisamment raisonné représente des risques. Pour rationaliser et rendre plus durable l'utilisation de ces traitements, des outils et des indicateurs devraient permettre de mieux cibler les troupeaux ou les lots et les périodes à risque et de mieux identifier les animaux à traiter.

Les Strongles Gastro-Intestinaux (SGI) des ruminants sont des vers ronds (nématodes) parasitant la caillette, l'intestin grêle ou le gros intestin. Leur cycle évolutif comprend une phase externe sur les pâtures (développement des œufs excrétés dans les fèces en larves infestantes L3) et une phase interne chez l'hôte (évolution des L3 ingérées avec l'herbe en larves L4, pré-adultes puis adultes qui se reproduisent et pondent des œufs). Ces parasites sont ubiquistes et on peut considérer que dès lors que les ruminants pâturent, ils s'infestent. Chez les bovins, il est communément admis que l'espèce la plus fréquente et la plus pathogène en régions tempérées est Ostertagia ostertagi, strongle digestif de la caillette (photo 1), tandis que chez les petits ruminants, les espèces les plus importantes sont Haemonchus contortus et Teladorsagia circumcincta dans la caillette et Trichostrongylus colubriformis dans l'intestin grêle.

Suite à des contacts prolongés avec ces parasites, les ruminants peuvent progressivement acquérir une immunité de type concomitante : immunité s'accompagnant d'une infestation persistante mais qui diminue fortement le taux d'installation des parasites (Claerebout et al 1998a). Cette immunité permet l'établissement d'un équilibre dynamique entre l'hôte et les parasites, et nécessite la présence du parasite pour perdurer (Vercruysse et Claerebout 1997). Cependant, le développement de l'immunité est très variable selon l'espèce de ruminant : il est très marqué chez les bovins et à l'inverse faible chez les caprins, alors que les ovins se positionnent de manière intermédiaire.

Les petits ruminants présentent ainsi une réceptivité et une sensibilité aux infestations par les nématodes gastrointestinaux plus importantes que les bovins. La chèvre en particulier développe une résistance aux réinfestations peu efficace (Hoste et al 2010) conduisant à des niveaux de parasitisme très élevés, notamment lorsqu'elles sont conduites au pâturage (moins vrai sur parcours). De surcroît, certains nématodes comme Haemonchus sp., absents chez les bovins en France métropolitaine, sont fréquemment rencontrés chez les petits ruminants et présentent un pouvoir pathogène particulièrement important (nématode hématophage de la caillette).

Chez les jeunes bovins non immuns, cette infestation peut engendrer des retards de croissance, voire des atteintes cliniques lorsque les charges parasitaires sont élevées (Armour et al 1979, Shaw et al 1998a et b). Chez les bovins adultes, l'expression clinique est très rare mais l'infestation peut induire des baisses de production (surtout étudiées chez les bovins laitiers) : le possible impact négatif de cette infestation sur la production laitière est largement documenté (Gross et al 1999, Sanchez et al 2004, Charlier et al 2009), mais les effets délétères sur les performances de reproduction restent plus équivoques (Charlier et al 2009).

De la même manière, les conséquences de l'infestation par les SGI chez les petits ruminants peuvent se traduire par des retards de croissance, des signes cliniques (diarrhées) voire de la mortalité chez les jeunes (Mandonnet et al 2003, Mahieu et al 2007) ainsi que des diminutions de production laitière, en particulier chez les animaux à haute production (Hoste et Chartier 1993).

Les mesures de contrôle de l'infestation par les SGI reposent essentiellement, parfois exclusivement, sur l'utilisation des traitements anthelminthiques ( $\mathrm{AH}$ ) qui éliminent les parasites chez l'hôte et préviennent des réinfestations lorsqu'ils sont rémanents (Hoste et al 2009, Charlier et al 2010a). Trois familles d'AH actifs contre les nématodes digestifs peuvent être utilisées : les benzimidazoles, le lévamisole, et les Lactones Macrocycliques (LM) (avermectines et milbémycines). Cette dernière famille domine le marché des AH chez les bovins (Sutherland et Leathwick 2011), notamment parce que ces molécules ont un spectre très large (endo- et ectoparasites), sont rémanentes (protection contre les réinfestations par O. ostertagi pendant 14 à 120 jours) et faciles à administrer (nombreuses formulations injectables et «pour-on») (Hoste et al 2009, Charlier et al 2010a). 
Chez les petits ruminants, à l'inverse, l'usage des benzimidazoles a été dominant en particulier chez les animaux laitiers en raison de délai d'attente nul pour le lait (Chartier et Hoste 1997). Cette situation est amenée à évoluer puisque, suite à de récentes et profondes révisions des temps d'attente, plus aucune spécialité à base de benzimidazoles ne dispose aujourd'hui de temps d'attente nul pour le lait.

Les médicaments strongylicides (qui tuent les strongles) sont administrés fréquemment, à l'ensemble des animaux d'une même catégorie d'âge, et souvent selon des protocoles de traitement invariables calés sur la conduite du troupeau : l'évaluation préalable du réel risque parasitaire s'appuyant sur des informations épidémiologiques et diagnostiques reste rare (Kenyon et Jackson 2012).

Cette synthèse vise $i$ ) à exposer les principaux risques associés à ce recours très fréquent et peu raisonné aux anthelminthiques pour lutter contre les SGI, et ii) à décrire des outils et indicateurs permettant de rationaliser l'usage de ces médicaments pour tout à la fois éviter l'expression clinique de l'infestation, sécuriser et optimiser les performances de productions et assurer la pérennité de ces molécules strongylicides.

\section{1 / Principaux enjeux de la rationalisation des traitements contre les strongles gastro- intestinaux chez les ruminants}

\section{1 / Apparition de résistances des parasites aux anthelminthiques}

La résistance à un anthelminthique (AH) donné dans une population parasitaire donnée correspond à l'augmentation de la fréquence des individus qui tolèrent des doses de cet $\mathrm{AH}$ supérieures à celles tolérées par les individus normaux, cette tolérance étant héréditaire (Sutherland et Leathwick 2011). Dans une population d'helminthes, il préexiste toujours une très faible proportion de vers présentant une aptitude génétique à résister à l'AH.

L'utilisation fréquente de molécules AH de la même famille exerce une forte pression de sélection sur les parasites (figure 1), et constitue ainsi un facteur de risque majeur d'émergence de populations de vers résistants (et cela particulièrement lorsque des LM rémanentes sont utilisées puisque l'avantage reproductif donné aux parasites adultes résistants chez l'hôte perdure dans le temps) (Leathwick et Besier 2014).

Photo 1. Bourse caudale d'Ostertagia ostertagi. Cette espèce vit dans la caillette et constitue l'espèce de strongle digestif la plus pathogène chez les bovins.

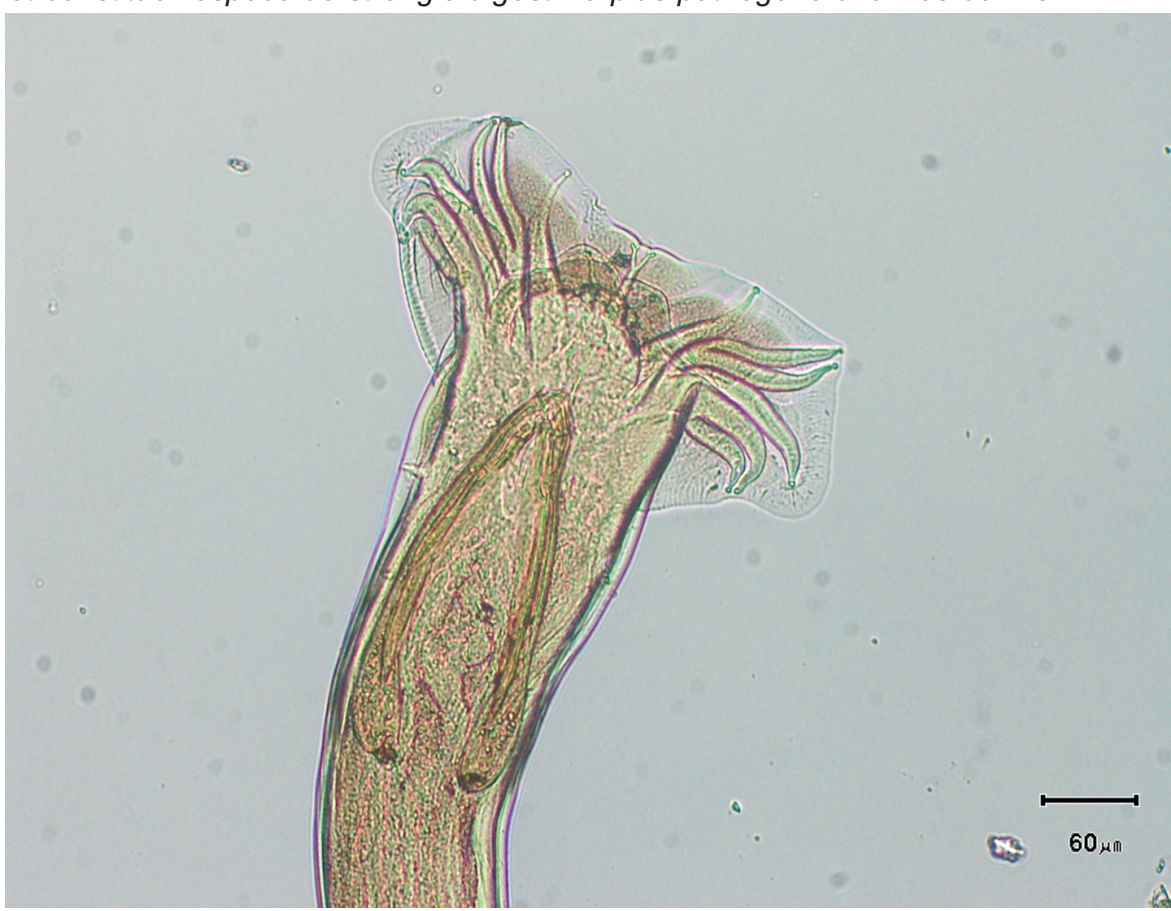

Le sous-dosage est aussi très souvent signalé comme facteur de risque d'apparition des résistances. Or, ce sous-dosage peut être fréquent chez les bovins en raison i) d'une estimation difficile du poids ; ii) d'une utilisation fréquente de molécules rémanentes dont la concentration diminue au cours du temps chez l'hôte jusqu'à atteindre une concentration létale pour les L3 au génotype sensible mais pas pour les L3 résistantes (Leathwick et Besier 2014) ; iii) d'une utilisation très fréquente des LM en formulation "pour-on», formulation associée à une grande variabilité de biodisponibilité entre individus (Gayrard et al 1999) pouvant mener à une sous-exposition des parasites à la molécule. De plus, ces formulations "pour-on » autorisent une diffusion de la molécule vers les animaux non traités (par léchage notamment) à des doses compatibles avec l'augmentation du risque de sélection de vers résistants (Bousquet-Mélou et al 2011).

Chez les petits ruminants, l'usage quasiexclusif et fréquent d'une même famille d'anthelminthique (les benzimidazoles), associé à un sous-dosage chez la chèvre (les posologies doivent être doublées chez les caprins par rapport aux ovins), sont considérés comme des facteurs essentiels du développement de la résistance des SGI aux benzimidazoles (Chartier et Hoste 1997).

Parmi les pratiques de traitement des ruminants, un autre facteur de risque majeur d'apparition de populations de SGI résistants aux AH est l'absence de conservation de population refuge de parasites (figure 1). Un refuge est une sous-population de parasites non soumise à l'action $\mathrm{AH}$ (donc non sélectionnée) lors d'un traitement: les stades libres présents sur les pâtures, les vers présents chez les hôtes non traités, et les stades larvaires inhibés vis-à-vis desquels l'AH n'est pas actif chez les hôtes traités (cas du lévamisole). Ces refuges permettent la dilution des gènes de résistance aux AH sélectionnés par le traitement en maintenant des gènes de sensibilité dans la population globale de parasites (van Wyk 2001, Kenyon et al 2009). Ce refuge sera d'autant plus réduit que le traitement concernera l'ensemble des animaux du lot, et sera administré à des périodes de faible infestivité des parcelles (sortie d'hiver, sécheresse, passage sur une nouvelle parcelle...) (figure 1). Or, ces pratiques de traitement sont fréquentes voire très fréquentes chez les ruminants.

Ces facteurs de risque majeurs d'émergence de résistances aux AH liés aux pratiques de traitements sont mieux connus chez les petits ruminants en raison des forts niveaux de résistances très largement répandues vis-à-vis des trois groupes de strongylicides (Jackson et Coop 2000, Kenyon et Jackson 2012, Rose et al 2015). La résistance aux anthelminthiques est ainsi un problème très sérieux chez les ovins et caprins dans le monde (existence de souches d'H. contortus résistantes à la quasi-totalité des $\mathrm{AH}$ existants), mais aussi en France quels que soient la région et le mode d'élevage. Les différentes enquêtes conduites dans notre pays (tableau 1) montrent des fréquences élevées de résistance à l'égard des benzimidazoles chez les ovins (viande et lait) et chez les caprins (Chartier et al 
Figure 1. Traitement anthelminthique, pression de sélection et populations refuges : émergence de populations de strongles gastro-intestinaux résistants aux molécules strongylicides.

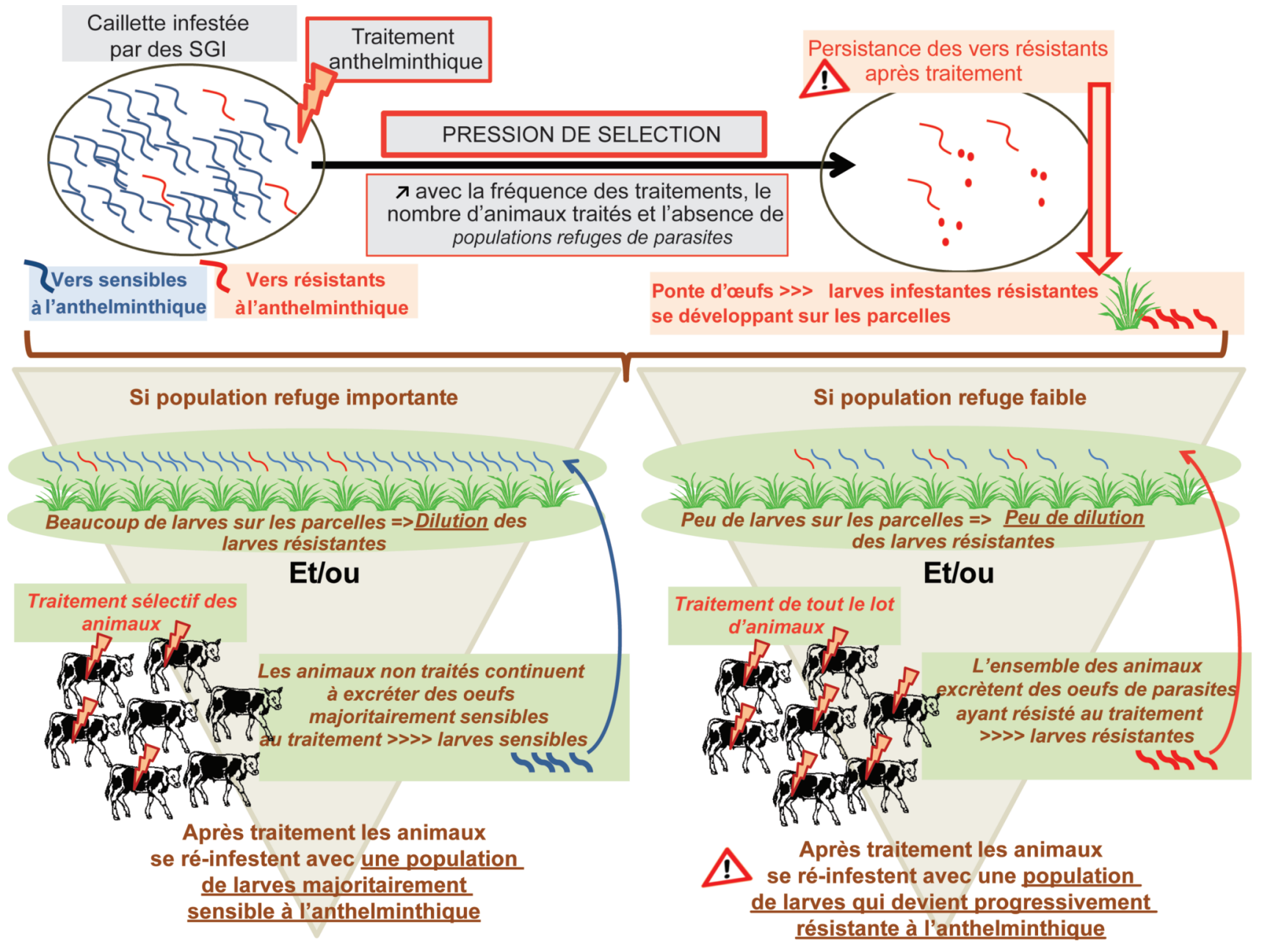

Tableau 1. Fréquence de la résistance des strongles gastro-intestinaux dans les élevages de ruminants en France selon la région et le type d'anthelminthiques (nombre d'élevages avec résistance/nombre total d'élevages enquêtés) (D'après Chartier et al 1998, Chartier et al 2001, Chartier et al 2007, Jacquiet et al 2014a, Paraud et al 2014, Geurden et al 2014, Geurden et al 2015).

\begin{tabular}{|c|c|c|c|c|}
\hline Espèces hôte & Région & Benzimidazoles & Lévamisole & $\begin{array}{c}\text { Lactones } \\
\text { Macrocycliques }\end{array}$ \\
\hline \multirow{6}{*}{ Ovins } & Val de Loire & $2 / 18$ & $1 / 18$ & n.e. \\
\hline & Deux-Sèvres & $19 / 23$ & $9 / 18$ & $0 / 21$ \\
\hline & Bretagne & $5 / 5$ & $2 / 5^{(1)}$ & $1 / 5^{(1)}$ \\
\hline & Aveyron & $5 / 5$ & n.e. & $0 / 5$ \\
\hline & $\begin{array}{l}\text { Pyrénées- } \\
\text { Atlantiques }\end{array}$ & $5 / 5$ & n.e. & $0 / 5$ \\
\hline & Loire & n.e. & n.e. & $1 / 1$ \\
\hline \multirow{5}{*}{ Caprins } & Deux-Sèvres & $15 / 15$ & n.e. & n.e. \\
\hline & Quercy & $15 / 18$ & $2 / 18$ & n.e. \\
\hline & Lyonnais & $10 / 10$ & n.e. & n.e. \\
\hline & France & n.e. & n.e. & $0 / 22$ \\
\hline & France & n.e. & $3 / 36$ & $6 / 36^{(1)}$ \\
\hline Bovins & Ouest & n.e. & n.e. & $3-5 / 8^{(2)}$ \\
\hline
\end{tabular}

n.e. : Non étudié $;^{(1)}$ : Suspicion ; ${ }^{(2)}$ : Résultats obtenus avec l'ivermectine et/ou la moxidectine. 
1998, Geurden et al 2014, Jacquiet et al 2014a), les parasites impliqués étant les SGI les plus fréquents : $T$. circumcincta, $T$. colubriformis et $H$. contortus. De manière plus sporadique, des signalements de bi-résistance benzimidazoles-lévamisole chez les caprins et de résistance à la moxidectine (lactones macrocycliques) chez les ovins ont été publiés (Paraud et al 2009, 2014).

Chez les bovins, la résistance des SGI a longtemps été sous-estimée, et les signalements de résistances aux $\mathrm{AH}$ sont moins fréquents et plus récents (Sutherland et Leathwick 2011). Mais ce phénomène ne doit plus être négligé chez les bovins. Depuis les années 2000, de nombreux cas documentés concernant les trois groupes de strongylicides ont été rapportés en Amérique du Sud, Australie, Nouvelle Zélande, et Etats-Unis (Sutherland et Leathwick 2011). Des résistances avérées ou des réductions d'efficacité des LM (faisant suspecter des résistances) ont aussi été mises en évidence en Europe : tout d'abord en GrandeBretagne (Stafford et Coles 1999), puis plus récemment en Allemagne, Belgique et en Suède (Demeler et al 2009). En France, l'existence de résistances aux anthelminthiques chez les bovins n'avait pas été signalée jusqu'à récemment. Cependant, une étude multicentrique menée en 2011-2012 en France, RoyaumeUni, Allemagne, et Italie a montré, parmi les huit troupeaux français impliqués, des baisses d'efficacité de l'ivermectine, de la moxidectine ou des deux molécules à la fois dans respectivement 3,5 et 3 troupeaux (Geurden et al 2015). Ces données sont les seules disponibles à ce jour en France pour les bovins.

Dans ces différentes études chez les bovins, Cooperia sp. (SGI de l'intestin grêle) reste le genre le plus fréquemment retrouvé après traitement. Deux éléments sont avancés pour expliquer la résistance plus fréquente dans cette espèce (Sutherland et Leathwick 2011) : d'une part, la plus faible sensibilité de ce parasite à l'égard des LM (« dose limiting species »), possiblement en lien avec des concentrations d'anthelminthiques atteintes dans l'intestin grêle inférieures à celle de la caillette, et, d'autre part, sa plus grande prolificité par rapport à $O$. ostertagi. Le pouvoir pathogène relativement faible de ce strongle digestif pourrait expliquer pourquoi l'augmentation de la prévalence des résistances aux AH ne s'est pas nécessairement accompagnée d'un signalement d'échecs de traitement visibles (pas d'augmentation de l'expression clinique des gastro-entérites parasitaires, ou d'augmentation des troubles zootechniques associés à l'infestation) (Sutherland et Leathwick 2011). Cependant, Ostertagia, SGI considéré comme le plus pathogène en zones tempérées, est aussi retrouvé parmi les vers résistants et cette résistance peut s'exprimer vis-àvis de l'un ou l'autre des trois groupes de strongylicides (Demeler et al 2009 , Sutherland et Leathwick 2011, Geurden et al 2015, Rose et al 2015). Des cas de résistances à plus d'un $\mathrm{AH}$ et pour plusieurs espèces de SGI ont été publiés (Sutherland et Leathwick 2011), ce qui suggère, en comparaison avec l'historique de ce phénomène chez les petits ruminants, que le développement de la résistance aux AH chez les SGI des bovins est probablement plus ancien que les signalements publiés ne le laissent penser (Sutherland et Leathwick 2011). Il y a cependant plus de signalements que d'enquêtes de prévalence véritables ce qui empêche d'avoir une vision précise de l'importance du phénomène (Kaplan et Vidyashankar 2012).

La ressource anthelminthique étant limitée et les délais de développement de nouveaux $\mathrm{AH}$ pouvant être très longs, il est illusoire d'envisager de répondre rapidement et efficacement à l'apparition des résistances avec l'utilisation de nouvelles molécules ayant un mode d'action différent. Cette situation doit inciter à la recherche et à la mise en place de méthodes de lutte aptes à ralentir voire à prévenir l'apparition des résistances aux $\mathrm{AH}$ chez les ruminants, à savoir : $i$ ) limiter le nombre de traitements anthelminthiques par une stratégie de ciblage des interventions, et $i i)$ préserver une population parasitaire refuge non soumise à la pression de sélection au niveau des stades larvaires ou des vers adultes. La prévention de l'apparition des résistances aux anthelminthique doit d'emblée faire partie de l'élaboration des plans de contrôle du parasitisme par les SGI.

\section{2 / Effets délétères des anthel- minthiques sur la faune non-cible}

Les résidus d'AH dans les matières fécales peuvent affecter des espèces noncibles, majoritairement des organismes invertébrés terrestres et aquatiques, et indirectement des vertébrés dont le régime alimentaire comprend de nombreux invertébrés (Lumaret et al 2012). L'impact environnemental des $\mathrm{AH}$ dépend de l'effet délétère exercé par la substance active (ou ses métabolites) sur le lieu de l'excréta, de la quantité de substance active excrétée, de la temporalité de l'excrétion et de la stabilité de ces résidus écotoxiques (McKellar 1997).

Les LM, et tout particulièrement les avermectines (ivermectine, doramectine, éprinomectine et abamectine), font partie des molécules les plus toxiques, notamment pour les organismes coprophages participant à la dégradation des bouses (bousiers). Les milbémycines (moxidectine) sont moins toxiques (Pritchard et al 2012) et les métabolites des benzimidazoles et du lévamisole s'avèrent relativement peu toxiques pour la faune coprophage (McKellar 1997, Lumaret et al 2012).

Les LM, qui ont un spectre d'activité très large comprenant insectes, acariens et nématodes, sont i) principalement excrétées dans les fèces, $i i)$ persistent dans l'organisme en subissant peu de biotransformations (pouvant ainsi être excrétées pendant plusieurs jours consécutifs sous leur forme active), et iii) sont faiblement biodégradables avec une forte affinité pour le sol et les matières organiques (McKellar 1997).

Par exemple, pour l'ivermectine, dans les 2 premiers jours après traitement, la concentration dans les fèces atteint un pic de $9,0 \mu \mathrm{g} / \mathrm{g}$ ou $3,9 \mu \mathrm{g} / \mathrm{g}$ de matière sèche après administration de $500 \mu \mathrm{g} / \mathrm{kg}$ en "pour-on 》 ou de $200 \mu \mathrm{g} / \mathrm{kg}$ par injection sous-cutanée, respectivement (Chiu et al 1990, Sommer et Steffansen 1993). Les bolus d'ivermectine, qui ne sont plus commercialisés en France aujourd'hui, entraînaient une excrétion fécale prolongée et une concentration élevée (concentration stable de $1,18 \mu \mathrm{g} / \mathrm{g}$ jusqu'à 120 jours post-traitement) (Alvinerie et al 1998). Or, il a été montré que dans des bouses provenant de bovins traités avec de l'ivermectine «pour-on », des effets larvicides sur l'espèce de bousier Aphodius constans sont possibles pendant 3 semaines après traitement avec une concentration létale médiane (CL50) de 0,5 à $0,8 \mu \mathrm{g} / \mathrm{g}$ de matière sèche (Lumaret et al 2007).

Plus généralement, les résidus fécaux des LM affectent peu les bousiers adultes matures, mais engendrent une mortalité accrue et des ralentissements de développement ovarien chez les jeunes adultes pendant plusieurs semaines après traitement des bovins (Lumaret et al 2012). Les larves sont en revanche extrêmement sensibles à ces résidus (jusqu'à 100\% de mortalité observée dans des bouses collectées dans les premières semaines après traitement), cette absence de développement larvaire pouvant perdurer pendant 105 jours lorsque des bolus d'ivermectine étaient administrés (Errouissi et al 2001).

Ces effets toxiques des LM peuvent largement impacter la dégradation des bouses sur les pâtures (Lumaret et al 2012), notamment en climat chaud et $\mathrm{sec}$ où les bousiers sont les organismes les plus importants dans ce processus de dégradation (Putman 1983). Ils colonisent rapidement les fèces, créent un réseau dense de galeries qui aèrent, fragilisent et dessèchent la masse stercorale. Ceci facilite l'entrée et l'activité des vers de terre qui poursuivent la dégradation 
(ces derniers jouant un rôle prédominant en conditions tempérées, Putman 1983). Ils stimulent de plus l'activité microbienne en inoculant les matières fécales avec les microorganismes qu'ils portent sur leur tégument. Les bousiers contribuent donc au recyclage des déjections en évitant leur accumulation au sol et la formation de refus. Ainsi, même si les LM ont peu voire pas d'effets toxiques sur les vers de terres (Lumaret et al 2012), leurs effets sur les insectes peuvent engendrer un ralentissement notable de la dégradation des bouses (Madsen et al 1990). Par exemple, dans une étude au cours de laquelle de l'ivermectine a été ajoutée dans des bouses à un niveau équivalent à celui observé dans les fèces de bovin traités, les bouses n'étaient pas dégradées après 340 jours, alors que les bouses «témoins » déposées au même moment et au même endroit étaient dégradées après 80 jours (Floate 1998). Cependant, d'autres études ont montré qu'il n'y avait pas de changement significatif dans la dégradation et l'accumulation des bouses sur les pâtures sur le long terme après utilisation de l'ivermectine (Barth et al 1993, 1994). Ces résultats contradictoires peuvent refléter la diversité de la faune coprophage entre pays (Lumaret et al 2012).

Cette écotoxicité et ses conséquences possibles sur la productivité des prairies en raison d'une mauvaise dégradation des fèces sont souvent négligées. Elles justifient aussi la rationalisation des traitements AH. Il faudrait $i$ ) limiter les traitements systématiques rémanents à large spectre (LM) qui impactent une faune non-cible essentielle au bon fonctionnement des écosystèmes pâturés (et surtout à la période de reproduction des insectes coprophages car leurs stades larvaires sont les plus sensibles : mai à août principalement en France), ii) favoriser le plus possible les traitements plus ciblés et de moindre impact.

\section{3 / Retard d'acquisition d'im- munité chez les jeunes bovins trop traités}

Cet effet adverse des AH sur le développement de l'immunité est particulièrement documenté chez les bovins en raison d'une forte expression de l'immunité vis-à-vis des strongles digestifs chez cet hôte.

Le développement de l'immunité anti-SGI chez les bovins dépend du temps de contact (durée d'exposition aux parasites) et de l'intensité de contact (challenge larvaire et charge parasitaire) avec les parasites (Vercruysse et Claerebout 1997). L'immunité vis-à-vis des espèces de l'intestin grêle s'installe assez rapidement : quelques mois suffisent pour observer une nette diminution de la quantité d'œufs excrétés dans les matières fécales, et un déclin des populations parasitaires chez l'hôte (Armour 1989). Il est communément admis qu'à l'issue de leur première saison de pâturage, les bovins ont développé une immunité protectrice efficace contre Cooperia et Nematodirus (Vercruysse et Claerebout 1997), et ces parasites sont retrouvés en très faible nombre chez les bovins adultes (Armour 1989). En revanche, pour les strongles de la caillette, Ostertagia notamment, l'acquisition de l'immunité est plus lente : ce n'est généralement qu'en fin de seconde saison de pâturage qu'une réduction des charges parasitaires est observable, ces parasites restant aussi présents, parfois en nombre non négligeable, chez les bovins adultes (Armour 1989).

Dans des études basées sur des infestations expérimentales avec Ostertagia (comparaison d'infestations continues et d'infestations tronquées par différents protocoles de traitement $\mathrm{AH}$ ), ont été observées : i) une diminution du taux d'installation des parasites de $65 \%$ après 24 semaines d'infestation continue (versus une diminution de seulement 28 et $54 \%$ lors d'infestation interrompue par un traitement avec un bolus d'ivermectine ou deux injections de doramectine, respectivement) (Claerebout et al 1998a) ; ii) une immunité bien développée après 21 semaines d'infestation continue ( $v s$ un faible développement de l'immunité après infestations tronquées au stade L3 ou L4) (Claerebout et al 1996). Dans les modèles biologiques développées par Grenfell et al (1987), la diminution du taux d'installation des parasites est évaluée à $50 \%$ après 150 jours de contact, $90 \%$ après 230 jours, et $99 \%$ après 320 jours.

Les traitements $\mathrm{AH}$, et particulièrement les molécules rémanentes ou les bolus délivrant une molécule strongylicide de manière continue ou séquentielle sur plusieurs semaines consécutives, réduisent la durée et/ou l'intensité de contact avec les parasites. Une utilisation importante des traitements AH chez les génisses de première saison de pâturage va ainsi conduire à un retard dans l'acquisition de l'immunité et donc à l'augmentation des traitements alors nécessaires chez les génisses de deuxième saison voire chez les adultes (Vercruysse et al 1994).

Cela a été confirmé dans plusieurs études de terrain lors d'infestations naturelles, en évaluant le parasitisme chez des génisses de seconde saison de pâturage en fonction de l'historique de traitement en première saison (Vercruysse et al 1994). Claerebout et al (1998b) ont notamment montré que la résistance à la réinfestation des génisses en seconde saison de pâturage était liée aux traitements AH antérieurs lors de leur première saison de pâturage, le développement de l'immunité étant inversement relié au degré de suppression du contact avec les parasites lors de cette première saison. De la même manière, il a été montré que l'infestation des vaches laitières (objectivée par la réponse en lait post-traitement anthelminthique) dépendait de l'immunité acquise avant le premier vêlage (évaluée par le temps de contact des génisses à l'herbe avec les parasites) (Ravinet et al 2014) (cf. infra). Il est probable que ce résultat soit extrapolable au cheptel allaitant. Or, les vaches allaitantes vêlant pour la première fois plus tardivement que les laitières, ces systèmes sont favorables à des temps de contact long avec les parasites et donc à l'acquisition d'une immunité solide avant vêlage. Ces considérations peuvent rendre très discutable le traitement $\mathrm{AH}$ des vaches allaitantes adultes.

Chez les génisses de renouvellement, l'usage des AH devrait donc être raisonné, en s'intégrant dans un contrôle des SGI visant à $i$ ) rechercher le contact avec les parasites pour favoriser le développement de l'immunité, et ii) maintenir des charges parasitaires à un niveau suffisamment bas pour éviter les conséquences zootechniques et cliniques de l'infestation.

\section{4 / Les stratégies de traitement permettant de maîtriser ces risques tout en assurant un contrôle efficace du parasitisme}

L'utilisation insuffisamment raisonnée (et dans bien des cas trop intensive) des AH pour lutter contre les SGI n'est donc plus acceptable. Tout en assurant un contrôle efficace de l'infestation permettant d'éviter les conséquences zootechniques et médicales, il est nécessaire de limiter l'usage des AH pour i) diminuer la pression de sélection (conservation de populations refuges), ii) répondre aux critères de l'agriculture durable préservant la biodiversité, et iii) ne pas entraver le développement de l'immunité (figure 2). Cette rationalisation des traitements est possible car l'impact des SGI sur les productions (et la santé) des ruminants est très variable entre troupeaux/lots, entre individus et entre saisons (Ravinet et al 2015a). En tenant compte de cette variabilité, les stratégies de traitement ciblé-sélectif visant au maintien d'une population parasitaire refuge permettent de répondre à ces objectifs de rationalisation car elles limitent les traitements AH (Kenyon et al 2009, Charlier et al 2014) : i) aux troupeaux/lots, et aux individus dans ces troupeaux/lots, qui pourront bénéficier du traitement en termes d'amélioration ou de maintien des 
Figure 2. Effets indésirables des anthelminthiques chez les ruminants et stratégies pour les limiter.

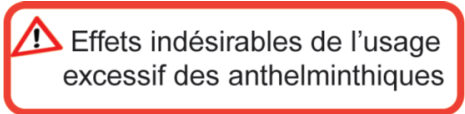

excessif des anthelminthiques
Stratégies pour limiter ces effets négatifs tout en assurant un bon contrôle du parasitisme chez les animaux

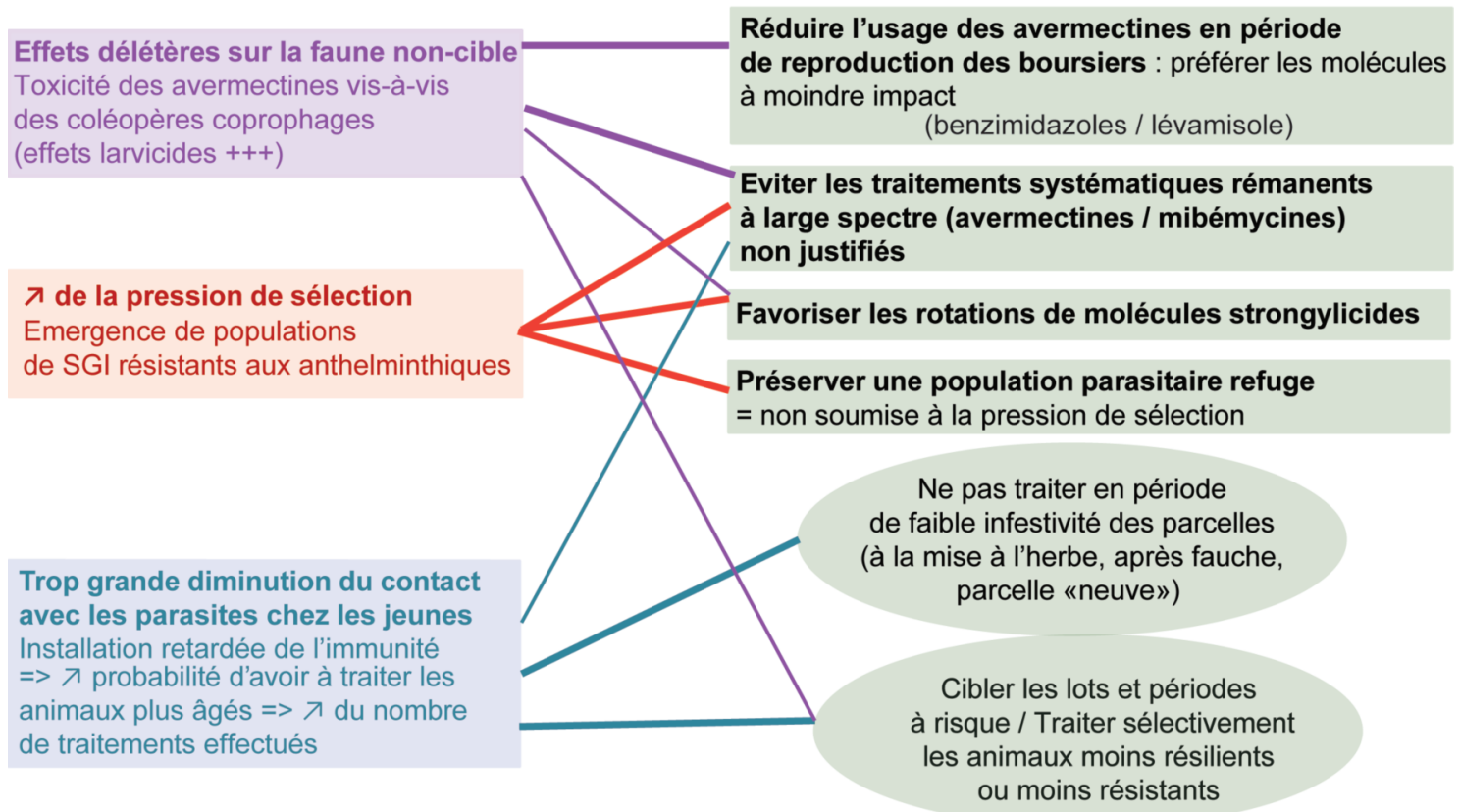

productions, ii) à la période à laquelle le risque parasitaire est avéré (exposition et niveaux d'infestation suffisants pour engendrer des pertes). Il s'agit donc de cibler les troupeaux/lots à risque et les périodes à risque, et de sélectionner les individus les plus parasités ou « souffrant $»$ le plus du parasitisme tout en gardant une population parasitaire refuge.

Le traitement ciblé (ciblage des troupeaux ou lots et ciblage des périodes à risque) doit idéalement prendre en compte la notion de risque pour le lot mais également la conservation d'une population refuge (stades larvaires présents sur les pâtures) au moment du traitement. Ainsi, le traitement ciblé doit s'appuyer en premier lieu sur la conduite de pâturage et l'infestivité des parcelles qui en résulte (parcelles unique ou en rotation, surfaces disponibles) (Chauvin et al 2012). Les facteurs météorologiques (température et humidité) doivent également être pris en compte car ils influent sur la vitesse de développement de l'œuf à la larve infestante, la migration des larves depuis les matières fécales et leur survie (Stromberg 1997) et par conséquent sur l'intensité de l'exposition des animaux aux parasites. Enfin, en particulier pour les bovins, le développement progressif de l'immunité doit être considéré puisqu'il conditionne la façon dont un lot d'animaux tolèrera l'exposition aux parasites. Par ailleurs, les traitements ciblés doivent éviter les périodes où les populations refuges sont particulièrement réduites. Cette approche doit amener à reconsidérer le traitement collectif des groupes d'animaux à la rentrée en bâtiment (la population refuge constituée par les stades larvaires sur les pâtures va décliner pendant l'hiver), pourtant pratiqué dans de nombreux élevages le plus souvent pour des raisons de praticité (cf. infra). Elle amène également à reconsidérer le traitement collectif d'animaux suivi d'un déplacement sur parcelle propre («dose and move ») (pas de refuge sur cette nouvelle parcelle non contaminée par les larves de SGI).

Le traitement sélectif vise à vermifuger les animaux les plus infestés (faible résistance) et/ou les plus sensibles (faible résilience), la population parasitaire refuge étant alors constituée des parasites hébergés par les animaux non traités. Ces animaux les plus infestés et/ou les plus sensibles sont le plus souvent les mêmes au cours de la saison ou d'une saison à l'autre (répétabilité) et l'on considère qu'il y a une base génétique (résistance innée ou acquise) à cette distinction (héritabilité) (Hoste et al 2002b). L'identification de ces animaux sous-entend la définition de critères pratiques, simples et peu onéreux avec des seuils de décision fiables. Idéalement, ces critères doivent pouvoir être appréhendés «au chevet» de l'animal et ne pas nécessiter de formation particulière de l'éleveur.

Pour mettre en œuvre les stratégies de traitement ciblé-sélectif, nous avons donc besoin de critères et d'outils fiables per- mettant de déterminer quand traiter et qui traiter, pour maîtriser l'infestation tout en maximisant les refuges.

Nous allons développer dans la suite de l'article les éléments disponibles en distinguant les petits ruminants et les bovins. En effet, bien que des outils puissent être similaires entre ces différentes productions, les très nombreuses particularités de chaque espèce (conduite d'élevage, sensibilité et réponse immunitaire de l'hôte, espèces parasitaires impliquées, épidémiologie et physiopathologie des infestations, résistance aux anthelminthiques) et la quantité de données disponible en petits ruminants rendent plus pertinente une présentation séparée. La situation rencontrée en élevage caprin laitier pâturant correspondant à la situation extrême en raison du cumul des difficultés rencontrées : faible résistance de l'hôte aux réinfestations, importance des nématodes résistants aux anthelminthiques (benzimidazoles) et forte limitation de l'arsenal thérapeutique en raison de longs temps d'attente pour le lait avec la majorité des molécules AH. Ainsi, la gestion des SGI en élevage caprin laitier peut être considérée comme un modèle illustrant les limites d'une gestion des SGI basée sur le " tout-anthelminthique », la nécessité d'évoluer vers une gestion raisonnée de ces infestations et enfin l'importance d'assurer la diffusion des résultats vers les éleveurs en les associant dans une coconstruction des démarches novatrices (cf. encadré 1). 
Encadré 1. La gestion des strongles gastro-intestinaux en élevage caprin laitier : comment sortir d'une situation d'impasse?

\section{1 / Complexité et limites de la gestion des Strongles Gastro-Intestinaux (SGI) par les anthelminthiques (AH) de synthèse en élevage caprin laitier}

Pour les consommateurs, l'élevage caprin laitier est associé à de nombreux signes de qualités ( $A O C, A O P$, IGP, AB). Le respect des cahiers des charges liés à ces labels suppose le plus souvent une part non négligeable d'exploitation du pâturage, ce qui signifie que les troupeaux seront soumis aux risques parasitaires helminthiques, et particulièrement aux infestations par les SGI.

Plus que chez les bovins et les ovins, le parasitisme par des SGI chez les chèvres laitières est une contrainte majeure qu'il faut pouvoir maîtriser pour 2 raisons :

- La faible aptitude des caprins à développer une réponse immunitaire pour réguler les populations de vers (Hoste et al 2010). Cela explique pourquoi des chèvres adultes, après plusieurs saisons de pâturage, restent à des niveaux d'infestations proches de ceux des primipares, ce qui n'est pas le cas chez les bovins et les ovins.

- L'importance des pertes de production provoquées (de 13 à $25 \%$ de baisse de production laitière chez les fortes productrices) en situation d'infestation subclinique (Hoste et Chartier 1993).

Cependant, c'est en élevage caprin laitier que la gestion des strongyloses gastro-intestinales est la plus complexe à mettre en œuvre, car soumise à de nombreuses contraintes :

- Les trois mêmes familles d'AHs sont utilisables chez les caprins comme chez les ovins et les bovins mais, en termes d'efficacité, des travaux en pharmacologie ont montré les spécificités caprines de métabolisation des $\mathrm{AH}$ et en conséquence la nécessité d'adapter les posologies (Chartier et Hoste 1997). Pour les firmes pharmaceutiques, la chèvre est considérée comme une espèce mineure en termes économiques. Par conséquent, i) les demandes d'AMM incluant l'espèce caprine ont été rares, et il est peu probable que des innovations thérapeutiques récentes (par exemple le monépantel) ou futures soient enregistrées pour les chèvres, ii) l'élevage caprin est parmi ceux où le principe d'application de la " cascade » pour emploi de molécules AH hors AMM est le plus répandu (Fresnay 2004).

- Dans les systèmes d'élevage caprins sous signes de qualité, la production de lait est liée à l'exploitation du pâturage et donc à l'exposition aux SGI. Jusqu'en 2015, seules trois molécules de la famille des benzimidazoles étaient sans délais d'attente pour commercialiser le lait. Les considérations économiques en découlant ont conduit les éleveurs à privilégier et surexploiter ces mêmes molécules pendant la lactation.

Au bilan, la forte sensibilité des caprins aux SGI, leurs particularités de métabolisation des xénobiotiques, et les restrictions réglementaires dans l'usage des traitements (temps d'attente pour le lait) ont conduit à une surutilisation des benzimidazoles, exerçant une forte pression de sélection sur les populations de SGI. II est donc peu étonnant que la prévalence des résistances aux AHs soit particulièrement forte en élevages caprins (Jackson et al 2012). En France, les enquêtes aléatoires datant du début des années 2000 mentionnent des prévalences supérieures à $80 \%$ pour les benzimidazoles (Chartier et al 1998, 2001). C'est ainsi le système de production où se sont révélées le plus rapidement les limites d'un mode de maîtrise monolithique reposant sur les seuls traitements de synthèse.

\section{2 / Nécessité fait loi : vers une gestion intégrée, durable des infestations par les SGI}

Au vu du constat précédent, l'élevage caprin laitier apparaît comme un des systèmes de production où, très tôt, le besoin d'une gestion intégrée des SGI est devenu un impératif. II faut souligner que ce type de gestion n'exclut pas les $\mathrm{AH}$ de synthèse mais vise à une utilisation plus pertinente.

Plusieurs projets de recherche récents ont eu pour objectif général d'explorer ces innovations dans la gestion du parasitisme par les SGI (projet CASDAR Parasitisme et projet STREP du métaprogramme INRA GISA) et, plus spécifiquement, d'identifier/ élaborer des indicateurs/outils permettant de répondre à deux questions clefs dans la décision ou non de traiter :

\section{Quand traiter?}

L'emploi de coproscopies de mélange (fondées sur des broyats de fèces provenant de 10 à 15 animaux) pour évaluer le niveau d'infestation d'un troupeau (ou de sous-groupes au sein d'un troupeau) a été validé (Manoloraki et al 2014). La réduction des coûts d'analyse en résultant devrait favoriser un suivi plus régulier des infestations par élevage et ainsi permettre de mieux identifier les périodes à risque.

\section{Qui traiter?}

Identifier les animaux les plus infestés au sein d'un troupeau est essentiel pour i) préserver directement leur santé et leur production, ii) réduire la contamination du pâturage et donc le risque pour les congénères, et pour iii) recourir à un minimum de traitements puisque l'on sélectionne les animaux à traiter individuellement. Plusieurs types de critères ont été retenus pour identifier les sujets " à risque » les plus infestés : 
- Des méthodes de laboratoire (ex : la coproscopie), mais leur coût et la logistique nécessaire les rend peu applicables en élevage.

- Des méthodes fondées sur l'observation de signes subcliniques : l'index FAMACHAC, l'index de diarrhée (DISCO) et la Note d'Etat Corporel (NEC) reflet de l'état général nutritionnel des animaux qui peut être affecté par la présence de vers (Bath and van Wyk, 2009). Ces divers critères reposent sur des grilles semi-quantitatives d'appréciation. Après un temps de formation, ils sont souvent facilement intégrés par les éleveurs. Cependant, toutes les variations de ces critères peuvent être d'origine multifactorielle et sont donc non spécifiquement liés au parasitisme par les SGI.

- Des critères fondés sur une réceptivité différenciée aux infestations : chez les caprins laitiers, une série d'études en conditions contrôlées puis en élevages ont montré que les primipares et les meilleures productrices au sein d'un troupeau présentaient souvent des niveaux d'excrétion plus élevés. Par comparaison aux traitements de l'ensemble du troupeau, des traitements sélectifs focalisés sur ces deux seules catégories se sont avérés sans conséquences néfastes sur la production et sur le niveau de parasitisme général du troupeau (Hoste et al 2002a).

\section{3 / De la validation scientifique des concepts de traitements ciblés-sélectifs vers leur adoption par les éleveurs}

Chez les petits ruminants, en système lait ou viande, les études explorant la validité de diverses modalités de traitement ciblésélectif afin de maîtriser le parasitisme par les SGI sont multiples (Kenyon et Jackson 2012).

Le dernier verrou à lever consiste à diffuser à grande échelle ce concept en élevages et favoriser son adoption par les éleveurs. L'importance de cette diffusion/adoption a depuis longtemps été identifiée (Van Wyk et al 2006). Elle nécessite : i) de comprendre les motivations des éleveurs pour essayer, accepter ou refuser des approches innovantes, puis ii) d'identifier les meilleurs circuits pour en assurer une large dissémination. II s'agit donc de confronter les données scientifiques aux critères des éleveurs.

Bath et van Wyk (2009) ont identifié une première liste d'étapes à prendre en compte dans cette démarche :

- Fournir des informations sur le parasitisme par les SGI, les AH, leurs limites actuelles, notamment les résistances aux $\mathrm{AH}$.

- Expliquer la validité scientifique de la démarche de traitement ciblé-sélectif (moins d'AH = frein au développement de résistances) ainsi que son efficacité dans la maîtrise des SGI.

- Compléter cette démonstration par des critères économiques : moins d'animaux traités = moins de coût direct $(\mathrm{AH})$ et moins de pertes en lait par application des délais d'attente.

- Prendre en compte les préoccupations des éleveurs : i) la simplicité d'utilisation des critères retenus, ii) le temps et la charge de travail.

- Impliquer les éleveurs dans le processus de décision. En ce domaine, une étude, entamée en 2013, est conduite pour appliquer les traitements sélectifs en élevages caprins dans le cadre d'une démarche participative en co-construction avec les éleveurs. Cette démarche vise à intégrer les spécificités de chaque élevage (ex : présence ou non $d$ 'H. contortus). Les résultats devraient être disponibles en 2018.

\section{2 / Outils pour le traitement ciblé-sélectif chez les petits ruminants}

\section{1 / Stratégie de traitement ciblé chez les petits ruminants}

De manière générale, la stratégie de traitement ciblé nécessitera d'apprécier :

- Les périodes à risque de fortes infestations, qui dépendent étroitement du type d'animaux à traiter et de leur sensibilité (chèvres, agneaux, brebis en gestation ou lactation), de leur mode de conduite et des conditions météorologiques locales, des populations de nématodes visées avec leur épidémiologie respective (Haemonchus sp, T. circumcincta, Nematodirus sp.,...),

- L'importance des populations larvaires sur les parcelles utilisées ou à utiliser au moment de l'intervention anthelminthique (appréciation de l'importance de la population refuge).
Pour apprécier le risque de forte infestation, la coproscopie (doublée le cas échéant d'une coproculture) peut être utilisée chez les petits ruminants, celleci pouvant être réalisée individuellement ou au niveau d'un lot d'animaux. En effet, chez les petits ruminants, le niveau d'excrétion d'œufs dans les fèces est assez bien corrélé à la charge parasitaire. Il n'existe pas de seuil unique d'interprétation quantitative, mais trois niveaux de valeurs coproscopiques ont toutefois été proposés par McKenna (1985) pour les ovins et les caprins et sont fréquemment utilisés :

$<500$ opg (faible), 500-2000 opg (moyen), > 2000 opg (fort) (opg = œufs par gramme de fèces).

Ces seuils ne sont que des indications et dépendent de la prolificité des parasites (élevée pour $H$. contortus et faible pour Nematodirus sp. par exemple). Ils doivent en outre être adaptés pour chaque situation (objectifs de production, limitations d'intrants). La coproscopie de mélange a été développée en petits ruminants et permet de déterminer avec fiabilité le niveau d'excrétion moyen d'un lot d'animaux. Elle s'appuie sur la réalisation de 10 à 15 prélèvements individuels qui seront mélangés ( $1 \mathrm{~g}$ par échantillon individuel) au laboratoire pour effectuer une seule analyse coproscopique quantitative de type McMaster (Jacquiet et al 2014b).

Pour apprécier l'importance de la population refuge, on sait que l'infestivité des parcelles est dépendante de leur mode d'utilisation et des conditions météorologiques qui prévalent localement. De manière générale, le froid de l'hiver et la sécheresse de l'été induisent de fortes mortalités dans les populations de larves infestantes, en particulier chez les petits ruminants où il n'y a pas l'inertie liée à l'effet bouse-réservoir existant chez les bovins. Ainsi, les traitements ciblés doivent éviter ces périodes où les populations refuges sont particulièrement réduites (traitement collectif à la rentrée, traitement puis déplacement sur parcelle saine). 


\section{2 / Stratégie de traitement sélectif chez les petits ruminants}

Les traitements sélectifs ayant fait l'objet d'études chez les petits ruminants s'appuient sur trois types d'indicateurs : indicateurs parasitologiques, cliniques ou liés à la production (Kenyon et al 2009).

\section{a) Indicateur parasitologique (indicateur de résistance de l'hôte)}

Cela consiste à mesurer directement l'excrétion fécale par coproscopie car, chez les petits ruminants, la mesure des opg est bien corrélée à la charge parasitaire pour Trichostrongylus $s p$. et Haemonchus sp. (un peu moins pour Teladorsagia et très peu pour Nematodirus $s p$.). Cependant, ce critère opg ne peut être aisément retenu pour la mise en œuvre du traitement sélectif en raison des délais et coûts d'analyse, de la manipulation des animaux et de la difficulté d'établir un seuil de traitement consensuel (par exemple plus de 300 opg pour les petits ruminants en Grèce ou une valeur supérieure à la moyenne du groupe pour les brebis laitières en Italie) (Kenyon et Jackson 2012).

\section{b) Indicateurs cliniques (indicateur de résilience de l'hôte)}

Plusieurs indicateurs physiopathologiques ont été étudiés chez les petits ruminants.

- FAMACHAC : cet indicateur s'appuie sur l'état d'anémie objectivé par l'examen de la muqueuse oculaire (photo 2) et noté de 1 à 5 (coloration décroissante) grâce à une carte plastifiée : les animaux présentant des notes $>3$ ou $\geq 3(30 \%$ des animaux dans certaines études) sont vermifugés (Bath et Van Wyk 2009). Cet indicateur a été développé dans les régions où $H$. contortus, strongle hématophage, constitue le strongle dominant comme les zones tropicales (cf. encadré 2). L'indicateur FAMACHAC semble plus difficilement applicable dans un contexte de polyparasitisme où $H$. contortus n'est pas toujours le strongle dominant dans chaque ferme et à chaque instant. Les résultats obtenus chez les ovins au Maroc montrent ainsi une bonne corrélation entre FAMACHA(C) et excrétion d'œufs de SGI tandis qu'en Italie, le traitement sélectif basé sur FAMACHAC aboutit à une infestation et un niveau de production laitière comparable aux groupes d'animaux non traités (Kenyon et Jakson 2012). Dans 10 troupeaux caprins en France dont 4 avec une présence significative d' $H$. contortus (65 à $95 \%$ des L3 en coproculture), les notes FAMACHAC ont montré une bonne corrélation avec l'hématocrite, mais très faible avec les opg

Photo 2. La technique FAMACHA® permet d'évaluer le niveau d'anémie chez les petits ruminants en lien avec l'infestation par Haemonchus contortus.

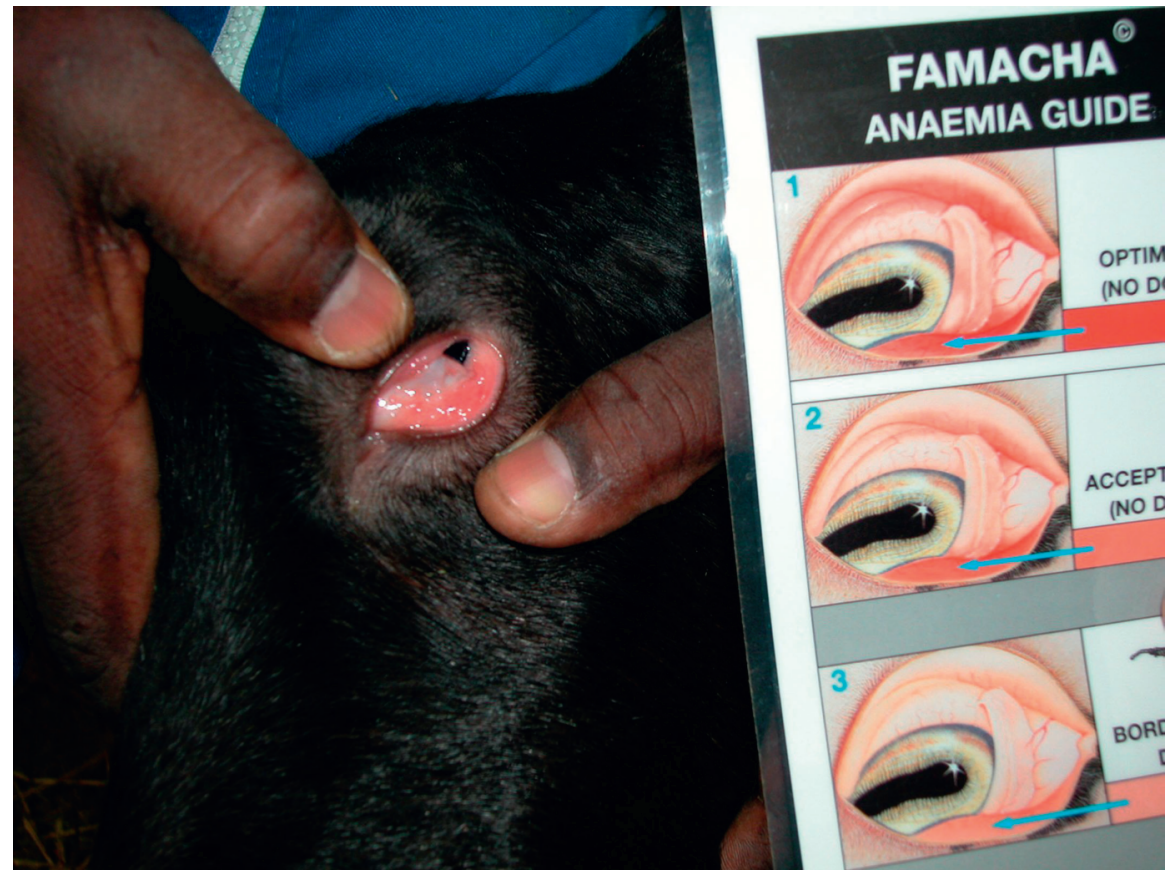

(corrélation entre FAMACHAC et opg fortement significative dans un seul troupeau, infesté essentiellement par Haemonchus et avec de forts niveaux moyens d'excrétion) (Kulo et al 2006). Dans une approche similaire dans 6 troupeaux caprins suisses, la corrélation globale entre FAMACHAC et opg n'a été significative que pour une date de prélèvement sur six ; en revanche, la corrélation spécifique avec les opg dus à Haemonchus (coprocultures individuelles) est globalement significative et une note $\geq 3$ permet de traiter valablement les animaux à plus de 300 opg dus à Haemonchus (Scheuerle et al 2010). Des données françaises en ovin (Cabaret 2004) confirment aussi ces limites.

- Index de diarrhée (DISCO) : 1'examen instantané des fèces permet l'attribution d'une note de 1 à 3 (liquide, mou, moulé). Ce caractère présente une bonne répétabilité et une bonne héritabilité. Il semblerait cependant plus adapté à l'évaluation du parasitisme de l'intestin grêle que de celui de la caillette (Cabaret 2004). Dans une étude chez des agneaux en Auvergne, 16 à $26 \%$ des animaux présentaient une note entre 1 et 2 mais avec un lien peu évident entre diarrhée et opg (Cabaret 2004). Inversement, l'indicateur DISCO a montré une bonne corrélation avec les opg chez des brebis au Maroc (traitement pour une note de 1 soit $15 \%$ des animaux) et chez des agneaux en Algérie (Kenyon et Jackson 2012).

- Index de souillure de l'arrière-train (DAGSCORE) : cet index s'appuie sur la répétition de diarrhée chez les ovins entraînant une souillure de l'arrière train. La relation opg-souillure est variable selon les études voire selon les saisons (au Royaume-Uni par ex. : relation positive en été mais pas au printemps). Cet indicateur très étudié en Australie semble trop tardif car s'accompagnant de pertes de production déjà installées (Kenyon et al 2009).

c) Indicateurs liés à la production (indicateurs de résilience)

- Gain moyen quotidien (GMQ) : ce critère peut être très intéressant pour le traitement sélectif car, chez les agneaux, la réduction de croissance est un signe très précoce avant l'apparition d'éventuels signes cliniques (Kenyon et al 2009). La valeur du GMQ comme critère de traitement sélectif a été en particulier étudiée dans les zones où $T$. circumcincta est l'espèce dominante (Europe du Nord). Plusieurs approches ont été testées : ne pas vermifuger les animaux les plus lourds lors du déparasitage (un refuge constitué par les $15 \%$ d'animaux les plus lourds semble efficace en termes de diminution de la pression de sélection), traiter les animaux en dessous d'un seuil de GMQ calculé (Kenyon et al 2009). L'utilisation de la variation de poids sur de courtes périodes de temps paraît prometteuse mais requiert un certain niveau d'équipement et d'automatisation (utilisé en Australie) : radioidentification des animaux, pesée automatisée, comparaison avec une croissance type, sélection des animaux avec portail électronique.

- Production laitière : la parité ou le niveau de production laitière des animaux pourrait être un critère valable de traitement sélectif car les chèvres primipares et les chèvres multipares à haute production (mesure avant la mise à 
Encadré 2. Chèvre Créole et Haemonchus contortus en Guadeloupe : apport de la méthode Famacha@.

Les caprins élevés au pâturage aux Antilles Françaises (Guadeloupe et Martinique) sont fortement affectés par le parasitisme gastro-intestinal dominé par les strongles Haemonchus contortus et Trichostrongylus colubriformis (Mandonnet et al 2003). En l'absence de contrôle de ces parasites, les pertes peuvent dépasser $50 \%$ du potentiel de production, en raison d'une mortalité importante des jeunes combinée à des performances diminuées pour les animaux survivant, même avec les populations locales, plutôt bien adaptées à ces contraintes (Mandonnet et al 2001). Le développement technique de l'élevage des petits ruminants, initié dans les années 1970, a reposé, entre autres, sur la prescription de traitements anthelminthiques à l'ensemble des animaux du troupeau, chaque mois pour les jeunes, tous les deux mois pour les adultes.

\section{Une généralisation des résistances aux anthelminthiques}

Bien que la plupart des éleveurs aient pratiqué des intervalles entre traitements plus variables et souvent plus longs que ce qui était recommandé, dès le milieu des années 1980 le premier cas de résistance au fenbendazole a été détecté en Martinique. Au milieu des années 1990 la résistance au fenbendazole était générale dans les élevages de Guadeloupe enquêtés. Une quinzaine d'années plus tard la situation est alarmante, avec 100\% des élevages ayant des populations de strongles digestifs résistants aux benzimidazoles, environ $80 \%$ résistants au lévamisole, $80 \%$ résistants à l'ivermectine et déjà $2 / 9$ résistants à la moxidectine (Mahieu et al 2014).

\section{Les traitements ciblés-sélectifs : la méthode Famacha@}

$H$. contortus étant l'espèce dominante, l'évaluation de l'anémie qu'il provoque par la méthode Famacha@ permet de piloter les traitements sélectifs des animaux adultes (Mahieu et al 2007). Cependant, pour des raisons pratiques, il reste souhaitable de continuer à traiter les jeunes de manière systématique tant qu'ils sont très sensibles aux strongles (vers 5-6 semaines, au sevrage, puis pendant les premiers mois après sevrage, en fonction de la pression parasitaire). Pratiquer des traitements ciblés-sélectifs (TCS) sur les adultes permet de maintenir une population parasitaire majoritairement sensible (Mahieu et al 2007) dans les parcelles qu'ils pâturent. En outre, il est souhaitable de faire pâturer les jeunes sevrés sur les mêmes parcelles que les adultes, en alternance, afin de bénéficier de l'effet dilution résultant du TCS chez les adultes ou, en d'autres termes, de gérer une seule population parasitaire à l'échelle de l'élevage entier (Mahieu et al 2015).

Dans ce contexte, la plupart des éleveurs ont pris conscience du risque de ne bientôt plus pouvoir disposer d'anthelminthique efficace et ils se montrent réceptifs au principe de TCS, d'autant plus qu'il s'accompagne d'une réduction des coûts vétérinaires, et qu'il est facile à mettre en pratique, sans investissement supplémentaire.

\section{Limites de la méthode Famacha@}

La méthode Famacha@ comme outil de pilotage des TCS est pertinente si $H$. contortus est le principal agent causal de l'anémie. C'est le cas aux Antilles, mais la situation est différente en Guyane, et plus largement partout où des hémopathogènes, comme Trypanosoma sp. ou Anaplasma sp., sont présents. Dans ce cas, la méthode Famacha@ ne peut pas permettre à elle seule de décider de l'application d'un traitement, elle devra être complétée par un diagnostic plus approfondi, et forcément plus lourd à mettre en œuvre. Par contre elle reste très utile pour l'éleveur dans la mesure où elle permet de détecter les animaux non anémiés, qui ne nécessitent aucun traitement, et elle facilite la détection des animaux devant faire l'objet de recherche d'hémopathogènes, puis de traitement spécifique.

l'herbe) présentent des niveaux d'infestation en moyenne plus élevés (Hoste et al 2002a). Le traitement sélectif appliqué sur ce principe concerne entre 48 et $66 \%$ du troupeau et a été validé dans 11 troupeaux en permettant d'obtenir un contrôle du parasitisme et une production laitière comparables à ceux d'un traitement de l'ensemble du troupeau (Hoste et al 2002b). En Grèce, le traitement sélectif des brebis ayant une production laitière journalière $>2 \mathrm{~L}$ permet une réduction du nombre de traitements de $80 \%$ par rapport à un double traitement collectif annuel, et cela sans engendrer des niveaux de parasitismes supérieurs ; en revanche, dans une étude italienne, le traitement des animaux ayant une production supérieure à la moyenne du groupe n'apportait pas de gain de production par rapport à des animaux non traités (Kenyon et Jackson 2012).
- Note d'état corporel (NEC) : la NEC (lombaire uniquement) variant de 1 à 5 a été testée chez la chèvre laitière en France (Paraud et al 2007). Cet indicateur est peu sensible car chutant uniquement lors de fortes infestations (1000 à 1800 opg en moyenne), de nombreuses variations pouvant de plus être liées à des facteurs non parasitaires (alimentation, stade de lactation et niveau de production). Dans cette étude, une note $\leq 2$ a permis de traiter $89 \%$ des fortes excrétrices ( $>1000 \mathrm{opg}$ ) tout en traitant également des animaux par excès. Ce même seuil a été utilisé chez la brebis en Grèce et a permis une réduction des traitements anthelminthiques de $50 \%$; chez les agneaux en croissance, la NEC s'est avérée trop peu sensible pour détecter les animaux les plus parasités (Kenyon et Jackson 2012).
De nombreux travaux ont été réalisés sur les indicateurs pour le traitement ciblé-sélectif des petits ruminants. Leurs principales limites sont rappelées dans l'encadré 3. D'autres approches basées sur le traitement aléatoire des animaux (traitement mensuel de 20\% des animaux $v s$ traitement mensuel collectif) semblent donner des résultats intéressants chez les ovins (Gaba et al 2012).

\section{3 / Outils pour le traitement ciblé-sélectif des génisses de renouvellement}

\section{1 / Les stratégies de traitement ciblé des génisses}

Pour mieux cibler les traitements chez les génisses, deux approches peuvent 
être considérées : i) chercher à bien identifier la période à risque en cours de saison de pâturage pour chaque lots considérés (prédire le risque pour évaluer la date optimale de traitement), ii) évaluer le risque à des dates "clé " (2-3 mois après la mise à l'herbe, à la rentrée en stabulation) pour évaluer si un traitement est nécessaire.

a) Identification des périodes à risque en cours de saison de pâturage

\section{- Informations à prendre en compte}

Les éléments relatifs à la conduite de pâturage, aux données météorologiques et à l'historique de contact avec les SGI (installation de l'immunité) doivent être pris en compte dans l'évaluation des périodes à risque chez les bovins pour savoir quand traiter (cf. $\S 1.4$ ).

\section{- Outils intégrant ces informations}

Des outils informatiques $i$ ) facilitant et standardisant la collecte et la saisie des informations relatives à la conduite de pâturage d'un lot de génisses, ii) intégrant les données de température et d'humidité et d'historique de contact avec les SGI, et iii) combinant ces informations pour prédire les périodes à risque ont été conçus et sont à la disposition des vétérinaires et des éleveurs. Ces outils sont basés sur des modèles qui miment le recyclage parasitaire (tableau 2). Chauvin et al (2015) ont notamment élaboré un système expert simulant l'accumulation des générations larvaires successives sur les parcelles pâturées (photo 3), et prédisant ainsi les périodes à risques pour les différents lots de génisses dont le planning de pâturage a été saisi. Cet outil peut être utilisé en troupeau laitier et en troupeau allaitant. Il intègre en effet une spécificité de l'élevage allaitant, à savoir le pâturage des veaux avec leur mère. Dans ces lots, la
Photo 3. Larve infestante de Cooperia oncophora (bovin).

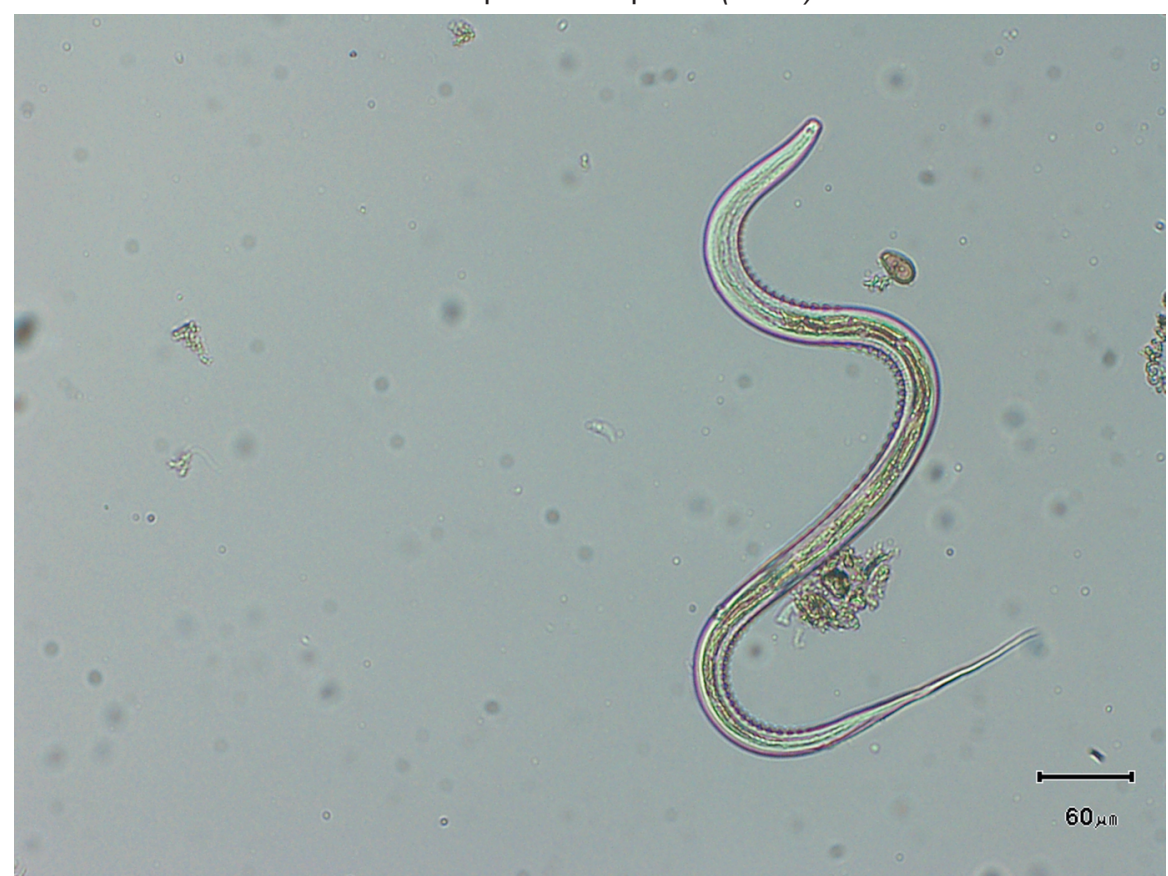

présence de mères immunisées peut diminuer la pression d'infestation pour les veaux (au moins en début de saison de pâturage) (Agneessens et al 1997), alors que les génisses laitières qui pâturent par groupe d'âge homogène ne peuvent bénéficier de ce phénomène lorsqu'elles sortent naïves pour la première fois au pâturage.

Ces différents outils ont, en outre, une valeur pédagogique puisqu'ils permettent de tester la pertinence et l'efficacité de plusieurs stratégies de traitement, et/ou de visualiser l'impact de la conduite de pâturage et des conditions météorologiques sur le risque parasitaire. Ils ont également une valeur opérationnelle puisqu'ils facilitent l'analyse du risque parasitaire et l'élaboration d'un plan de contrôle adapté aux contraintes d'un élevage donné. L'idéal serait de vérifier les prédictions de ces modèles avec des examens de laboratoires (coproscopie, dosage de pepsinogène sérique ou ELISA Ostertagia) qui, dès le milieu de la première saison de pâturage, peuvent être de bons reflets de l'exposition aux parasites ou du niveau d'infestation (Eysker et Ploeger 2000) (cf. § 3.1.b). Le comptage larvaire sur prélèvement d'herbe pourrait être adapté à ce suivi du risque puisqu'il permet une évaluation directe du niveau de contamination des pâtures par les larves infestantes (Grüner et Raynaud 1980, Eysker et Ploeger 2000). Cependant, cet examen est très peu utilisé sur le terrain car il présente de nombreux inconvénients : lourdeur de la procédure de prélèvement d'herbe, grande fragilité du prélèvement, délais prélèvement-analyse devant être très courts, rareté des laboratoires effectuant le comptage larvaire, incertitude de mesure probablement élevée.

Encadré 3. Les limites des indicateurs pour le traitement ciblé-sélectif chez les petits ruminants.

1. La plupart des indicateurs sont liés à la résilience et sont donc modérément corrélés aux OPG. II en résulte un contrôle partiel de la contamination de l'environnement.

2. De nombreux indicateurs impliquent la manipulation des animaux, ce qui peut être une contrainte sérieuse dans les grands troupeaux.

3. Ces indicateurs présentent tous des limites en termes de spécificité (autres causes d'anémie qu'Haemonchus sp., origine multiple d'un faible niveau de production ou d'un état corporel faible).

4. Les indicateurs cliniques peuvent être considérés comme trop tardifs car alors les pertes de production sont probablement déjà importantes.

5. Chaque indicateur doit être accompagné d'un seuil, celui-ci dépendant de très nombreux facteurs et devant être raisonné au plus près de l'exploitation et de ses caractéristiques.

6. L'association de plusieurs indicateurs apporte en général un faible gain et représente une charge de travail peu soutenable. 
Tableau 2. Outils d'aide à la décision pour évaluer quand traiter un lot de génisses au pâturage contre les strongles gastrointestinaux : différents outils informatiques prédisant les périodes à risque.

\begin{tabular}{|c|c|c|c|c|c|c|}
\hline \multirow[b]{2}{*}{ Outil } & \multirow[b]{2}{*}{ Modélisation } & \multicolumn{4}{|c|}{ Prise en compte de : } & \multirow{2}{*}{$\begin{array}{l}\text { Résultat de la } \\
\text { prédiction du } \\
\text { risque }\end{array}$} \\
\hline & & $\begin{array}{l}\text { la conduite } \\
\text { de pâturage }\end{array}$ & $\begin{array}{c}\text { la } \\
\text { température }\end{array}$ & l'humidité & $\begin{array}{l}\text { l'installation } \\
\text { de l'immunité }\end{array}$ & \\
\hline $\begin{array}{l}\text { Simulateur } \\
\text { du risque } \\
\text { parasitaire } \\
\text { (version } \\
\text { simplifiée } \\
\text { online - site } \\
\text { web Idele, et } \\
\text { version } \\
\text { complète } \\
\text { tableur à } \\
\text { Oniris) }\end{array}$ & $\begin{array}{l}\text { Modélisation } \\
\text { mathématique } \\
\text { de la durée } \\
\text { d'évolution des } \\
\text { parasites sur } \\
\text { les pâtures et } \\
\text { des dates } \\
\text { d'apparition } \\
\text { des } \\
\text { générations } \\
\text { successives } \\
\text { de larves } \\
\text { infestantes. } \\
\text { Système- } \\
\text { expert } \\
\text { simulant un } \\
\text { raisonnement } \\
\text { du risque. }\end{array}$ & $\begin{array}{l}\text { Ou } \\
\text { Choix d'une } \\
\text { conduite de } \\
\text { pâturage } \\
\text { "type » } \\
\text { dans un } \\
\text { menu } \\
\text { déroulant }\end{array}$ & $\begin{array}{l}\text { OUI } \\
\text { Température } \\
\text { journalière } \\
\text { moyenne de la } \\
\text { commune la } \\
\text { plus proche de } \\
\text { l'élevage } \\
\text { ou d'une } \\
\text { "grande } \\
\text { zone » (Brest, } \\
\text { Rennes, } \\
\text { Limoges, } \\
\text { Grenoble...) }\end{array}$ & $\begin{array}{l}\text { Modélisation } \\
\text { d'un effet } \\
\text { "sécheresse " } \\
\text { : arrêt de la } \\
\text { migration des } \\
\text { larves hors des } \\
\text { bouses et } \\
\text { reprise lors du } \\
\text { retour des } \\
\text { pluies (risque } \\
\text { parasitaire } \\
\text { élevé après la } \\
\text { sécheresse) }\end{array}$ & $\begin{array}{l}\text { OUI } \\
\text { Génisses } \\
\text { considérées } \\
\text { immunisées } \\
\text { après } 240 \\
\text { jours de } \\
\text { contact effectif } \\
\text { avec les } \\
\text { larves } \\
\text { infestantes de } \\
\text { SGI }\end{array}$ & $\begin{array}{l}\text { Sous forme de } \\
\text { frise temporelle } \\
\text { colorée : } \\
\text { - vert = absence de } \\
\text { risque = nombre de } \\
\text { générations } \\
\text { larvaires insuffisant } \\
\text { pour engendrer des } \\
\text { pertes } \\
\text { - rose au rouge = } \\
\text { période à risque } \\
\text { parasitaire croissant } \\
=\text { nombre de } \\
\text { générations } \\
\text { larvaires croissant et } \\
\text { augmentant } \\
\text { progressivement le } \\
\text { risque parasitaire }\end{array}$ \\
\hline $\begin{array}{l}\text { Eva3P } \\
\text { (Merial) }\end{array}$ & $\begin{array}{l}\text { Modélisation } \\
\text { mathématique } \\
\text { de l'ensemble } \\
\text { du cycle } \\
\text { parasitaire. }\end{array}$ & $\begin{array}{l}\text { Partiellement } \\
\text { (succession } \\
\text { de parcelles) }\end{array}$ & $\begin{array}{l}\text { OUI } \\
\text { Température } \\
\text { journalière } \\
\text { moyenne de } \\
\text { grandes } \\
\text { régions }\end{array}$ & $\begin{array}{l}\text { OUI } \\
\text { variation du } \\
\text { taux de } \\
\text { migration des } \\
\text { larves des } \\
\text { bouses vers la } \\
\text { pâture et } \\
\text { lessivage des } \\
\text { larves lors de } \\
\text { fortes pluies } \\
\text { d'automne. }\end{array}$ & $\begin{array}{l}\text { OUI } \\
\text { évolution } \\
\text { décroissante } \\
\text { du taux } \\
\text { d'installation } \\
\text { des parasites } \\
\text { en fonction du } \\
\text { temps de } \\
\text { contact avec } \\
\text { les larves } \\
\text { infestantes de } \\
\text { SGI }\end{array}$ & $\begin{array}{l}\text { Sous forme de } \\
\text { graphiques : } \\
\text { courbes décrivant, } \\
\text { en fonction du } \\
\text { temps, le nombre } \\
\text { de larves sur les } \\
\text { parcelles, l'excrétion } \\
\text { des œufs, le } \\
\text { nombre de parasites } \\
\text { chez les bovins et le } \\
\text { nombre de larves en } \\
\text { hypobiose. }\end{array}$ \\
\hline
\end{tabular}

\section{(1) : Groupement de Défense Sanitaire.}

L'utilisation de ces outils informatiques modélisant le risque parasitaire permet de bien répondre théoriquement aux objectifs du contrôle des SGI chez les génisses : $i$ ) identifier les périodes favorables à l'installation de l'immunité sans danger pour les animaux (contact avec les parasites mais pression d'infestation faible), ii) identifier les périodes à risque pour le lot de génisses, périodes au cours desquelles les parasites doivent être supprimés par traitement ciblé pour maintenir la croissance et bien sûr pré- venir l'expression clinique de l'infestation. Une telle rationalisation des traitements via ces outils pourrait permettre de réduire de moitié le nombre de traitements administrés aux génisses (Chauvin et al 2015).

b) Ciblage des lots de génisses à risque à des dates "clé »

Deux stratégies ont été particulièrement étudiées, le traitement des génisses à la rentrée en bâtiment à l'automne ou le traitement environ 2 à 3 mois après la mise à l'herbe.

- Évaluer si un traitement est nécessaire à la rentrée

À la rentrée en stabulation des génisses (surtout les génisses de première saison de pâturage), l'examen de choix pour évaluer l'intérêt d'un traitement $\mathrm{AH}$ et estimer rétrospectivement si le plan de contrôle de l'infestation a été efficace au cours de la saison de pâturage est le 
dosage de pepsinogène sérique (Kerboeuf et al 2002). Le pepsinogène est le précurseur de la pepsine, enzyme protéolytique de la caillette. Le taux de pepsinogène sérique (exprimé en milli-unités de tyrosine, mUTyr) est un marqueur des lésions de la caillette et un indicateur de la charge parasitaire en $O$. ostertagi hébergée par les jeunes bovins (Kerboeuf et al 1981).

Pour mettre en œuvre cet examen, 5 à 10 prélèvements sanguins doivent être effectués sur des génisses d'un même lot, c'est-à-dire ayant le même historique de pâturage et de traitement $\mathrm{AH}$. La méthode de dosage couramment utilisée dans les laboratoires français est la méthode enzymatique INRA (Kerboeuf et al 2002). Cependant, certains laboratoires utilisent une méthode développée en Belgique un peu différente (Dorny et Vercruysse 1998), donnant des valeurs plus élevées et avec des seuils d'interprétation différents. Pour une interprétation correcte des résultats obtenus, il est donc primordial de se renseigner sur la méthode employée (et ses normes d'interprétation) dans le laboratoire.

Individuellement, des animaux porteurs d'une charge parasitaire équivalente peuvent présenter des concentrations de pepsinogène variables (Kerboeuf et al 1981, 2002). L'interprétation du dosage de pepsinogène sérique devra donc se faire à l'échelle du lot, à partir de la moyenne des taux de pepsinogène individuels (Kerboeuf et al 1981, 2002). Si, à l'issu de la 1ère saison de pâturage, des valeurs élevées (autour de 2000 mUtyr) indiquent qu'un traitement $\mathrm{AH}$ est nécessaire, des valeurs trop basses proches des normes (300-600 mUTyr) indiquent que le contrôle a été trop drastique en cours de saison et n'a pas permis un contact avec les parasites favorisant l'installation de l'immunité.

Cependant, d'autres approches, explorées récemment, permettraient d'éviter le recours aux examens de laboratoire à la rentrée en stabulation pour identifier les lots de génisses à traiter. Ainsi, la prise en compte d'indicateurs simples sur la saison de pâturage écoulée (période de mise à l'herbe des génisses, durée de pâturage et niveau de supplémentation) permet de différencier à la rentrée en stabulation des niveaux moyens d'infestation de groupes de génisses (Merlin et al 2016a). Dans ce travail, l'association d'une sortie des génisses avant le mois de juin, d'une durée de pâturage de plus de 198 jours et d'une absence de supplémentation a permis de caractériser les lots les plus exposés aux SGI (niveaux élevés de pepsinogène en fin de saison) dans lesquels la croissance était impactée par le parasitisme (Merlin et al 2016a).
De même, l'apparition de la troisième génération larvaire (indicateur généré par le simulateur de risque parasitaire vu plus haut (tableau 1) (Chauvin et al 2015) permet une très bonne discrimination $a$ posteriori des lots de génisses laitières les plus infestés (22 lots sur 24) et représente donc un outil intéressant d'évaluation des lots à risque sans recours aux examens de laboratoire (Merlin et al 2016b).

Le traitement à la rentrée, très pratiqué sur le terrain, est bien documenté mais est très discutable puisqu'il s'accompagne d'une pression de sélection importante en raison de l'absence de préservation d'une population refuge (diminution hivernale de la taille de la population de stades larvaires présents sur les pâtures). Ceci explique les travaux plus récents sur une approche de traitement sélectif, notamment à cette période de l'année de rentrée des animaux, le refuge étant alors assuré par la présence de parasites chez les animaux non traités (cf. § 3.3).

\section{- Évaluer si un traitement est nécessaire en cours de saison de pâturage}

L'identification des lots de génisses à traiter peut s'effectuer 2 à 3 mois après la sortie des animaux par coproscopie. La coproscopie 8 semaines après la mise à l'herbe a ainsi été proposée pour prédire si l'infestation resterait au stade subclinique ou s'exprimerait cliniquement en cours de saison de pâturage (valeurs moyennes obtenues $>200$ opg) (Shaw et al 1997, 1998b). Les concentrations moyennes de pepsinogène sérique d'un lot mesurées à la même période semblent être un bon prédicteur de la croissance et des manifestations cliniques de fin de saison de pâturage (Ploeger et al 1994, Shaw et al 1997). Cette approche permet de s'assurer de l'existence d'une population refuge importante puisque les animaux sont traités alors qu'il y a effectivement des stades larvaires sur les parcelles.

\section{3 / Les stratégies de traitement sélectif des génisses}

a) Le gain moyen quotidien (GMQ) comme critère de traitement sélectif des génisses

Une première étude (Höglund et al 2009) théorique (preuve de concept) a montré qu'il serait envisageable de baser une stratégie de traitement sélectif sur le GMQ calculé 4 à 8 semaines après la mise à l'herbe (traitement sélectif des génisses avec un GMQ $<0,75 \mathrm{~kg} / \mathrm{j}$ ). Une étude de terrain a ensuite été menée (Höglund et al 2013) pour tester une telle stratégie : à partir de 8 semaines après la mise à l'herbe, traitement sélectif (doramectine injectable) des génisses présentant un GMQ inférieur à la médiane des GMQ d'un lot de génisses non infestées (car sous traitement anthelminthique rémanent continu). Le lot d'animaux soumis à cette stratégie sélective a été comparé à i) un lot d'animaux jamais traités, et ii) au lot d'animaux non infestés. À la rentrée en stabulation, la croissance moyenne des génisses du lot traité sélectivement $(0,36-0,50 \mathrm{~kg} / \mathrm{j})$ restait inférieure à celle des génisses non infestées $(0,39-0,61 \mathrm{~kg} / \mathrm{j})$, mais était supérieure à celle des génisses non traitées (0,23-0,42 kg/j) (Höglund et al 2013). Cependant, ce résultat est insuffisant pour attester de l'efficacité ou non de ces stratégies sélectives. En effet, dans cette étude, la stratégie sélective a été comparée à deux pratiques de traitement « extrêmes » (absence totale de traitement ou traitements répétés tous les mois). Pour évaluer l'apport d'une telle stratégie, il faudrait donc aussi la comparer aux pratiques courantes, appliquées par les éleveurs sur le terrain (1 à 2 traitements de tout le lot pendant la saison de pâturage).

Une étude récente conduite en France sur des génisses laitières a montré qu'en fin de première saison de pâturage, le GMQ individuel était corrélé négativement aux indicateurs parasitaires (en particulier le niveau d'anticorps antiOstertagia, exprimé en Ratio de Densité Optique $=$ RDO) dans les groupes d'animaux moyennement ou fortement exposés aux SGI (Merlin et al 2016a). Dans la prolongation de ce travail (Merlin et al 2016b), l'étude de la sensibilité et la spécificité de différents seuils de GMQ à la rentrée pour détecter différents niveaux de parasitisme (approche ROC) a montré, dans les conditions du suivi, qu'un compromis était obtenu avec un seuil de GMQ de $683 \mathrm{~g} / \mathrm{j}$ pour un RDO Ostertagia de 0,93 . Ce seuil de GMQ $(<0,683 \mathrm{~g} / \mathrm{j})$ permet de détecter $76 \%$ des animaux à RDO $>0,93$ (sensibilité) tandis que près d' 1 animal sur 2 est au-dessus du seuil de GMQ tout en ayant des RDO $<0,93$ (spécificité de $56 \%$ ). Selon les objectifs poursuivis (traiter le plus d'animaux parasités ou ménager une taille suffisante de refuge), différents seuils de GMQ avec leurs caractéristiques sont proposés (Merlin et al 2016b). Le développement de stratégies intégrant le critère GMQ doit donc se poursuivre car ces résultats sont prometteurs (photo 4). De plus, même si cet indicateur n'est pas spécifique du parasitisme par les SGI (d'autres facteurs non parasitaires peuvent avoir un impact sur la croissance), il constitue un bon « indicateur compromis » entre résistance (capacité de l'hôte à réguler la charge parasitaire et réduire l'excrétion) et résilience (capacité de l'hôte à moins pâtir des effets pathogènes des parasites et maintenir leurs performances). 
Photo 4. Pesée en plein champ de génisses laitières de première saison de pâturage.

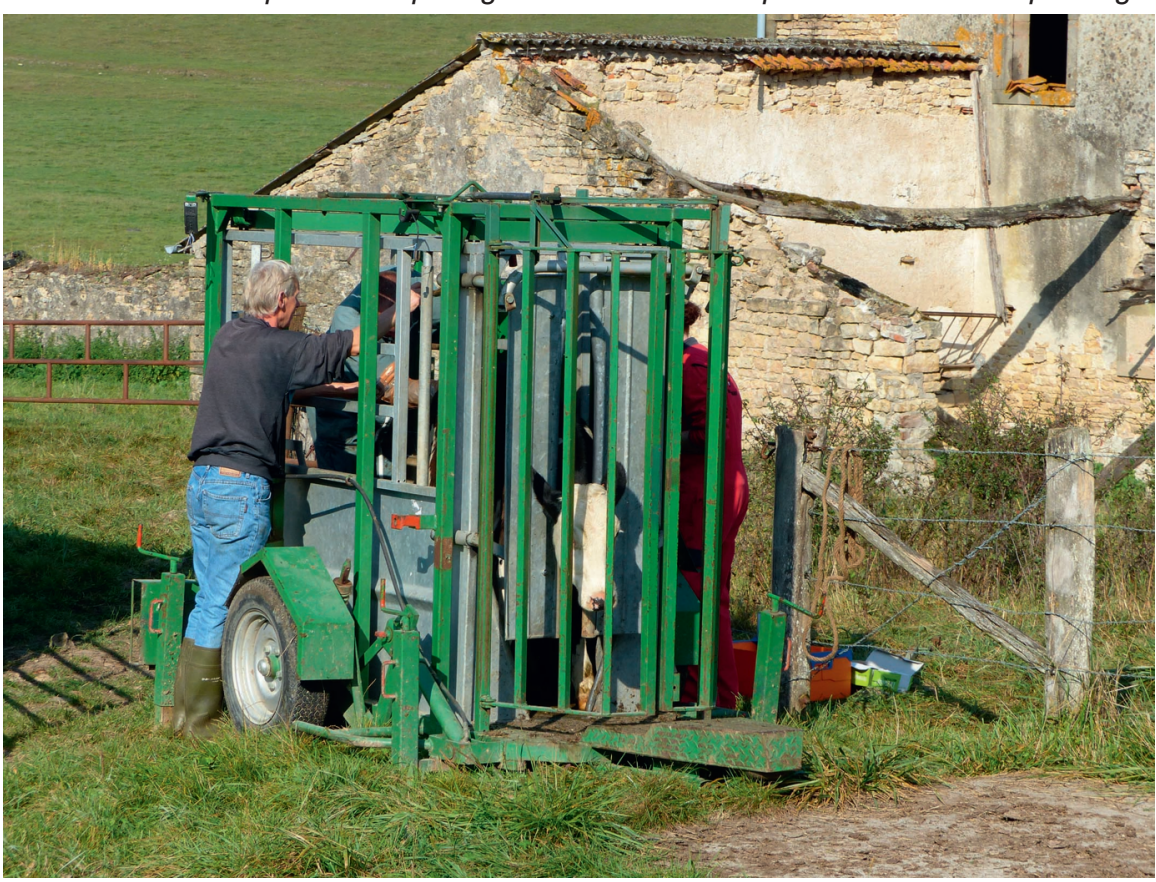

b) Traitement sélectif des génisses basé sur des indicateurs parasitaires

Dans une étude irlandaise récente (O’Shaughnessy et al 2015), une stratégie de traitement sélectif basée sur des indicateurs parasitaires (et intégrant aussi les strongles respiratoires) a été testée : traitement sélectif (ivermectine injectable) des génisses excrétant des larves de dictyocaule (strongle respiratoire), et/ou présentant un taux de pepsinogène sérique élevé $(\geq 2$ UTyr, méthode de dosage différente de la méthode INRA) associée à un résultat coproscopique $\geq 200$ opg (méthode de MacMaster) (ces examens étant effectués toutes les 3 semaines). Le lot de génisses soumis à cette stratégie a été comparé à un lot traité 3 fois à l'ivermectine (jours 0,42 et 84). Les performances de croissance ont été similaires dans ces deux groupes, et le nombre de traitements a été réduit de $50 \%$ dans le groupe traité sélectivement. Ces résultats sont très intéressants et montrent qu'une stratégie de traitement sélectif intégrant SGI et strongles respiratoires est possible. La stratégie testée reste cependant très peu opérationnelle car elle nécessite de prélever tous les animaux (sang et matières fécales) toutes les 3 semaines.

\section{4 / Outils pour le traitement ciblé-sélectif des vaches}

Cette partie sera dédiée aux vaches laitières car $i$ ) les travaux sur le sujet ont essentiellement été conduits en troupeaux laitiers, $i$ i) les traitements strongylicides sont souvent conseillés chez les vaches laitières car il a été démontré à maintes reprises que ces parasites peu- vent avoir un impact négatif sur la production laitière, iii) les animaux vêlant tôt en système laitier, et les génisses étant souvent traitées, il est probable qu'un certain nombre de vaches entrant en phase de production n'aient pas pu développer une immunité protectrice satisfaisante vis-à-vis des SGI.

\section{1 / Effets du traitement anthel- minthique sur la production laitière}

L'effet d'un traitement $\mathrm{AH}$ sur la Production Laitière (PL) des vaches a été étudié depuis plusieurs décennies. La réponse en lait post-traitement est très variable entre études, et souvent d'amplitude modérée $(<1 \mathrm{~kg} / \mathrm{vache} / \mathrm{j})$. Pour 75 études publiées entre 1972 et 2002, l'effet du traitement varie entre $-2,17$ et $+3,16 \mathrm{~kg} / \mathrm{vache} / \mathrm{j}$, le gain de PL posttraitement est positif dans près de $80 \%$ de ces études, mais significatif dans seulement $53 \%$, et a été estimé par métaanalyse à globalement $+0,35 \mathrm{~kg} / \mathrm{vache} / \mathrm{j}$ (Sanchez et al 2004). Plus rarement, une chute significative de production laitière post-traitement est rapportée (Tharaldsen et Helle 1989, Ravinet al 2016). De plus, l'effet du traitement sur la PL est très variable entre troupeaux et entre individus (Ploeger et al 1989, 1990 , Sanchez et al 2004, Vanderstichel et al 2013, Ravinet et al 2014, Verschave et al 2014).

Il apparaît donc que le traitement $\mathrm{AH}$ n'induit pas systématiquement un gain de PL, que la réponse en lait moyenne à attendre est modérée, et que le traitement des vaches adultes ne se justifiera que dans des contextes, des élevages et sur des individus préalablement identi- fiés comme forts « contributeurs 》 dans ces réponses en lait moyennes modérées. Ceci souligne d'autant plus l'importance des stratégies de traitement ciblé-sélectif chez ces bovins laitiers adultes.

\section{2 / Identification de la période optimale de traitement des vaches}

Le traitement du troupeau de vaches à la rentrée en stabulation est une pratique assez courante. L'effet du traitement sur la PL a déjà été largement étudié à cette période. Une augmentation de production laitière peut être constatée, mais les résultats ne sont pas toujours significatifs, et l'effet demeure souvent modéré (Ploeger et al 1989, 1990, Charlier et al 2007, Charlier et al 2010b, Ravinet et al 2014).

Le traitement des vaches contre les SGI en cours de saison de pâturage est une pratique moins courante. Cette période de traitement présenterait l'intérêt d'autoriser la conservation d'une population refuge de parasites plus importante qu'à l'automne (présence des stades libres sur les parcelles pâturées). À cette période, l'effet du traitement sur la production laitière est moins bien documenté et les résultats sont contradictoires. Une étude rapporte un effet positif mais dans un échantillon de très faible taille $(n=40$ vaches) (Forbes et al 2004). Des protocoles de traitements rémanents (éprinomectine) répétés toutes les 4 à 6 semaines peuvent aussi induire une augmentation de production laitière (Gibb et al 2005), mais ces stratégies ne sont pas applicables et pas recommandables sur le terrain car en opposition avec l'objectif de rationalisation du recours aux $\mathrm{AH}$. Une étude indique que l'effet du traitement sur la production laitière n'est pas différent au pâturage et en période de stabulation (Verschave et al 2014), mais dans d'autres travaux récents (Ravinet et al 2016), une chute marquée de production laitière a été observée après traitement au fenbendazole administré 2 mois après la mise à l'herbe $(-0,92 \mathrm{~kg} / \mathrm{vache} / \mathrm{j})$.

Dans l'attente de pouvoir disposer de plus de données sur l'effet du traitement $\mathrm{AH}$ en cours de saison de pâturage, il conviendrait, par prudence, de plutôt cibler ces traitements à la rentrée en stabulation des vaches (sauf en cas d'épisode de bronchite vermineuse au pâturage), et d'accorder alors une grande importance au traitement sélectif dans les troupeaux préalablement ciblés pour assurer une population parasitaire refuge chez les vaches non traitées.

\section{3 / Identification des troupeaux à cibler à la rentrée en stabulation}

Pour identifier les troupeaux de vaches dans lesquels le traitement $\mathrm{AH}$ 
pourrait entraîner une augmentation de production laitière, deux indicateurs sont apparus prometteurs, notamment lorsqu'ils sont pris en compte simultanément: le niveau d'anticorps antiOstertagia dans le lait de tank (mesuré par technique ELISA et exprimé en Ratio de Densité Optique - RDO), et le Temps de Contact Effectif (TCE) avec les larves infestantes de SGI avant le premier vêlage.

Le niveau d'anticorps anti-Ostertagia dans le lait de tank (RDO lait de tank) est considéré comme un reflet de l'exposition moyenne du troupeau de vaches en lactation aux SGI. Il est en effet associé à des niveaux d'exposition élevés à la pâture (Charlier et al 2005, Forbes et al 2008), et une corrélation significative entre niveaux d'anticorps antiOstertagia (mesurés sur 5 vaches du troupeau et moyennés) et nombre de larves présentes sur les pâtures a été mise en évidence (Eysker et al 2002). Plusieurs études ont mis en évidence une réponse en lait post-traitement $\mathrm{AH}$ à l'automne tendant à être meilleure dans les troupeaux à RDO lait de tank élevé (Charlier et al 2007, Ravinet et al 2014). Cependant, ces résultats manquent de significativité statistique et sont parfois équivoques (Charlier et al 2007). Cela suggère que le RDO lait de tank ne devrait pas être pris en compte seul dans le processus d'identification des troupeaux à traiter de manière ciblée. Il pourrait être complété d'un indicateur reflétant le statut immunitaire du troupeau de vaches, puisque le risque parasitaire lié aux SGI dépend de manière générale de i) l'amplitude de l'exposition aux parasites, et de ii) la capacité des animaux à « résister » à l'infestation (Chauvin et al 2012). Or, il n'existe pas de dosage biologique évaluant l'immunité acquise chez les bovins. On sait cependant que l'installation de cette immunité dépend largement de la durée de contact avec les SGI (cf. supra). Dans un programme de recherche récent (CASDAR parasitisme), Ravinet et al (2014) ont donc élaboré un indicateur visant à refléter le statut immunitaire du troupeau de vaches sur la base d'informations relatives à l'historique de pâturage et de traitement des animaux. Il s'agit du Temps de Contact Effectif (TCE) des génisses avec les larves infestantes de SGI avant le premier vêlage. Ce TCE correspond à la durée de la (des) saison(s) de pâturage des génisses à laquelle il faut soustraire les périodes au cours desquelles le contact avec les larves est annulé (traitement rémanent) ou amoindri (sécheresse avec forte complémentation) (Ravinet et al 2014).

À l'automne, dans un échantillon de 25 troupeaux du nord-ouest de la France $(n=1088$ vaches), une réponse en lait post-traitement positive et significative a été observée dans les troupeaux à TCE bas $(<8$ mois pour tout ou partie des primipares entrant dans le troupeau adulte), alors qu'il n'y avait pas d'effet du traitement sur la production laitière dans les troupeaux à TCE élevé $(>8$ mois pour toutes les primipares) (Ravinet et al 2014). Il a de plus été montré que la réponse en lait était meilleure dans les troupeaux caractérisés par un TCE bas et un RDO lait de tank élevé (comparée à la réponse en lait dans les troupeaux uniquement caractérisés par un TCE bas ou un RDO lait de tank élevé) (Ravinet et al 2015b). Le TCE serait donc un facteur de variation de la réponse en lait d'autant plus discriminant que l'exposition du troupeau aux SGI est élevée. Ces résultats soulignent l'intérêt de combiner plusieurs indicateurs dans le processus d'identification des troupeaux où les SGI peuvent entraver la production laitière, et pourraient être à la base de la construction d'un arbre décisionnel facilitant le ciblage des traitements $\mathrm{AH}$. Cette approche doit cependant être validée et affinée pour notamment déterminer les seuils de TCE et de RDO associés à la plus forte probabilité de réponse en lait post-traitement.

\section{4 / Les critères pour sélectionner les vaches à traiter}

Pour trouver des critères individuels permettant d'identifier les vaches dont la production est améliorée après traitement, les variations individuelles de la réponse en lait en fonction de différents indicateurs, parasitaires ou zootechniques, ont été étudiées.

\section{a) Traitement sélectif basé sur des indi- cateurs parasitaires}

Quelques études ont suggéré qu'une réponse en lait positive peut être attendue pour les vaches présentant un niveau élevé d'anticorps anti-Ostertagia (ELISA effectué sur un prélèvement de lait individuel en fin de lactation, et réponse en lait positive lors de la lactation suivante après traitement au vêlage) (Sanchez et al 2002, Vanderstichel et al 2013). Cependant, Charlier et al (2010b) ont souligné que la valeur de cet indicateur reste médiocre pour prédire la réponse en lait post-traitement, et Verschave et al (2014) ont observé des réponses en lait positives aussi chez des vaches avec des niveaux d'anticorps bas. Par ailleurs, Ravinet et al (2014) ont montré que le gain de production laitière post-traitement était meilleur chez les vaches présentant un niveau d'anticorps faible dans le sérum. Les résultats sont donc contradictoires, ce qui limite l'utilisation de cet indicateur sur le terrain. De plus, ce critère serait peu opérationnel puisque son utilisation nécessiterait un prélève- ment et une analyse de laboratoire par vache pour évaluer s'il faut la traiter ou pas.

La coproscopie et le taux pepsinogène sérique ne seraient pas non plus de bons critères de traitement sélectif puisqu'ils ne sont pas des facteurs de variation discriminants de la réponse en lait posttraitement (Ravinet et al 2014).

\section{b) Traitement sélectif basé sur des indi- cateurs zootechniques}

Souvent, le niveau de production calculé sur les performances de la lactation précédente n'est pas un facteur de variation discriminant de la réponse en lait post-traitement (Ploeger et al 1990, Ravinet et al 2014).

Pour ce qui est du stade de lactation au moment du traitement, il a été observé à plusieurs reprises que les vaches répondaient mieux au traitement lorsque celuici est administré dans la première moitié de la lactation (Sanchez et al 2004, Charlier et al 2010b, Ravinet et al 2014).

La réponse en lait était liée à la parité dans plusieurs études. Cependant, alors que quelques études ont montré des réponses meilleures chez les primipares (Forbes et al 2004, Ravinet et al 2014), d'autres études ont observé au contraire que les multipares répondaient mieux (Charlier et al 2010b, Verschave et al 2014). Finalement, Charlier et al (2014) indiquent que l'âge en soi n'influencerait pas la réponse en lait, mais qu'elle serait liée à des facteurs reflétant la durée de contact avec les parasites (historique de pâturage et de traitement anthelminthique des vaches).

En considérant la valeur des différents critères étudiés, un profil de traitement ciblé-sélectif opérationnel combinant des caractéristiques de la vache et du troupeau dans lequel elle évolue pourrait être : traitement à la rentrée en stabulation des jeunes vaches, plutôt en début de lactation, dans les troupeaux à TCE faible et RDO lait de tank élevé. Ce profil est le reflet d'un défaut possible d'immunité chez les jeunes vaches en début de lactation (puisque provenant de troupeaux à TCE faible), qui sont soumises à une pression d'infestation probablement élevée (RDO lait de tank élevé à la rentrée en stabulation). Une stratégie de traitement ciblé-sélectif basée sur ce profil serait donc fondée sur le plan biologique, mais elle doit être validée dans des travaux ultérieurs.

\section{Conclusion}

L'usage raisonné des anthelminthiques est une condition essentielle à la durabilité du contrôle de l'infestation par les 
SGI. Pour la première fois, dans une note de réflexion sur la résistance aux anthelminthiques parue en juillet 2016, l'Agence Européenne des Médicaments (EMA) recommande explicitement de baser les traitements anthelminthiques sur la mise en évidence préalable d'une infestation par un outil de diagnostic approprié. Cette recommandation rappelle les obligations de certains cahiers des charges en agriculture biologique où seul le traitement curatif, après établissement du diagnostic, est autorisé. La règlementation peut aboutir à une réduction d'usage comme ce fut le cas par exemple au Danemark où l'obligation de prescription pour les anthelminthiques à partir de 1999 s'est accompagnée pour les chevaux d'un plus large recours à la coproscopie et au traitement sélectif (Nielsen 2009), (en France, les anthelminthiques en production animale ne sont délivrés que sur ordonnance).

Les outils et les indicateurs décrits dans cet article s'appuient selon le cas sur des critères de résistance ou de résilience aux infestions par les SGI ou sur l'estimation de risque et présentent d'importantes limites telles que leurs performances (sensibilité, spécificité) et leur faisabilité pratique (coût, temps). Les travaux doivent ainsi se poursuivre sur la recherche d'indicateurs à la fois plus fiables, plus aisés et moins coûteux. Pour autant, les imperfections des outils actuels ne doivent pas nous dissuader de leur usage car seules les démarches de réduction d'usage des anthelminthiques, par le traitement ciblé et le traitement sélectif, et prenant en compte le maintien d'une population refuge, sont de nature à réduire le risque ou l'extension de la résistance aux anthelminthiques.

Cependant, les nouveaux outils et indicateurs permettant cette rationalisation ne pourront être adoptés sur le terrain que si les freins à l'acceptation de nouvelles pratiques sont dépassés, et plus encore lorsque ces pratiques innovantes sont en contradiction avec des habitudes anciennes de traitement. Cela nécessite de comprendre ces freins, et de communiquer de manière pertinente auprès des éleveurs et des vétérinaires prescripteurs. En poursuivant et en renforçant les collaborations entre parasitologues, vétérinaires praticiens, conseillers en élevage des organismes agricoles et industrie pharmaceutique, un discours commun pourra être construit et délivré sur le terrain, se basant sur des preuves scientifiques, et mettant en exergue la nécessité et les possibilités de rationalisation des traitements.

\section{Remerciements}

Cette synthèse a été écrite suite à des travaux sur les strongles gastro-intestinaux chez les ruminants conduits dans deux UMT : l'UMT Maîtrise de la Santé des Troupeaux Bovins à Oniris et l'UMT Santé des Petits Ruminants à l'ENVT. Les auteurs remercient les programmes ou organismes suivants pour le financement de ces travaux exposés dans cet article : le programme GISA STREP de l'Inra, le programme CASDAR du Ministère de l'Agriculture, de l'Agroalimentaire et de la Forêt (projet Casdar parasitisme ${ }^{\circ} 1127$ ), et la chaire Agriculture Ecologiquement Intensive (Agrial, Terrena, Triskalia, Agrocampus Ouest, ESA, Oniris). Les auteurs remercient tous les éleveurs ayant participé à ces travaux pour leur accueil, leur aide et leur coopération.

\section{Références}

Agneessens J., Dorny P., Hollanders W., Claerebout E., Vercruysse J., 1997. Epidemiological observations on gastrointestinal nematode infections in grazing cow-calf pairs in Belgium.Vet. Parasitol., 69, 65-75.

Alvinerie M., Sutra J.F. Galtier P., Lifschitz A. Virkel G., Sallovitz J., Lanusse C., 1998. Persistence of ivermectin in plasma and faeces following administration of a sustained-release bolus to cattle. Res. Vet. Sci., 66, 57-61.

Armour J., 1989. The influence of host immunity on the epidemiology of Trichostrongyle infections in cattle. Vet. Parasitol., 32, 5-19.

Armour J., Bairden K., Duncan J.L., Jennings F.W., Parkins J.J., 1979. Observations on ostertagiasis in young cattle over two grazing seasons with special references to plasma pepsinogène levels. Vet. Rec., 105, 500-503.

Barth D., Heinze-Mutz E.M., Roncalli R.A., Schlüter D., Gross S.J., 1993. The degradation of dung produced by cattle treated with an ivermectin slow-release bolus. Vet. Parasitol., $48,215-227$

Barth D., Heinze-Mutz E.M., Langholff W., Roncalli R.A., Schlüter D., 1994. Colonisation and degradation of dung pats after subcutaneous treatment of cattle with ivermectin or levamisole. Appl. Parasitol., 35, 277-293.

Bath G.F., Van Wyk J.A., 2009. The Five Point Check (C) for targeted selective treatment of internal parasites in small ruminants. Small Rum. Res., 86, 6-13.

Bousquet-Mélou A., Jacquiet P., Hoste H., Clément J., Bergeaud J.P., Alvinerie M., Toutain
P.L., 2011. Licking behaviour induces partial anthelminthic efficacy of ivermectin «pour-on» formulation in untreated cattle. Int. J. Parasitol., 41, 563-569.

Cabaret J., 2004. Parasitisme helminthique en élevage biologique ovin : réalités et moyens de contrồle. INRA Prod. Anim., 17, 145-154

Chiu S.H.L., Green M.L., Baylis F.P., Eline D., Rosegay A., Meriwether H., Jacob T.A., 1990. Absorption, tissue distribution, and excretion of tritium-labeled ivermectin in cattle, sheep, and rat. J. Agr. Food Chem., 38, 2072-2078.

Charlier J., Claerebout E., De Mûelenaere E., Vercruysse J., 2005. Associations between dairy herd management factors and bulk tank milk antibody levels against Ostertagia ostertagi. Vet. Parasitol., 133, 91-100.

Charlier J., Duchateau L., Claerebout E, Vercruysse J., 2007. Predicting milk-production responses after an autumn treatment of pastured dairy herds with eprinomectin. Vet. Parasitol., $143,322-328$

Charlier J., Höglund J., Samson-Himmelstjerna G., Dorny P., Vercruysse J., 2009. Gastrointestinal nematode infections in adult dairy cattle: Impact on production, diagnosis and control. Vet. Parasitol., 164, 70-79.

Charlier J., Demeler J., Höglund J., von SamsonHimmelstjerna G., Dorny P., Vercruysse J., 2010a Ostertagia ostertagi in first-season grazing cattle in Belgium, Germany and Sweden: general levels of infection and related management practices. Vet. Parasitol., 171, 91-98.

Charlier J., Vercruysse J., Smith J., Vanderstichel R., Stryhn H., Claerebout E., Dohoo I., 2010b.
Evaluation of anti-Ostertagia ostertagi antibodies in individual milk samples as decision parameter for selective anthelminthic treatment in dairy cows. Vet. Parasitol., 93, 147-152.

Charlier J., Morgan E.R., Rinaldi L., van Dijk J., Demeler J., Höglund J., Hertzberg H., Van Ranst B., Hendrickx G., Vercruysse J., Kenyon F., 2014. Practices to optimise gastrointestinal nematode control on sheep, goat and cattle farms in Europe using targeted (selective) treatments. Vet. Rec., 175, 250-255.

Chartier C., Hoste H., 1997. La thérapeutique anthelminthique chez les caprins. Le Point Vétérinaire, 28, 1907-1914.

Chartier C., Pors I., Hubert J., Rocheteau D. Benoit C., Bernard N., 1998. Prevalence of anthelmintic resistant nematodes in sheep and goats in Western France. Small Rum. Res., 29, 33-41.

Chartier C., Soubirac E., Pors I., Sivestre A., Hubert J., Couquet C., Cabaret J., 2001. Prevalence of anthelmintic resistance in gastro intestinal nematodes of dairy goats under extensive management conditions in South Western France. J. Helminthol., 75, 325-330

Chartier C., Pors I., Rehby L., Kerboeuf D. 2007. Anthelmintic resistance survey in French dairy goat nematodes in four main breeding areas. In: Proc. Int Conf. World Assoc. Adv. Vet. Parasitol. (WAAVP), 19-23 August, 2007. Gent, Belgique, p213.

Chauvin A., Ravinet N., Chartier C., 2012. Nouvelles approches du contrôle des strongyloses gastro-intestinales. Le Point Vétérinaire, $43,14-21$. 
Chauvin A., Ravinet N., Vermesse R., 2015. Development of a simulation model of the parasitic risk related to gastrointestinal nematode infection in grazing heifers. In: Proc. Int. Conf. World Assoc. Adv. Vet. Parasitol. (WAAVP), Liverpool, UK, p. 197.

Claerebout E., Hilderson H.,Meeus P., De Marez T., Behnke J., Huntley J., Vercruysse J., 1996. The effect of truncated infections with Ostertagia ostertagi on the development of acquired resistance in claves. Vet. Parasitol., $66,225-239$.

Claerebout E., Vercruysse J., Dorny P., Demeulenaere D., Dereu A., 1998a. The effect of different infection levels on acquired resistance to gastrointestinal nematodes in artificially infected cattle. Vet. Parasitol., 75, 153-167.

Claerebout E., Dorny P., Vercruysse J., Agneessens J., Demeulenaere D., 1998 b. Effects of preventive anthelmintic treatment on acquired resistance to gastrointestinal nematodes in naturally infected cattle. Vet. Parasitol., 76, 287-303.

Demeler J., Van Zeveren A.M.J., Kleinschmidt N., Vercruysse J., Höglund J., Koopmann R., Cabaret J., Claerebout E., Areskog M., Samson-Himmelstjerna G., 2009. Monitoring the efficacy of ivermectin and albendazole against gastrointestinal nematodes of cattle in Northern Europe. Vet. Parasitol., 160, 109-115.

Dorny P., Vercruysse J., 1998. Evaluation of a micro method for the routine determination of serum pepsinogen in cattle. Res. Vet Sci., 65, 259-262.

Errouissi F., Alvinerie M., Galtier P., Kerbeouf D., Lumaret J.P., 2001. The negative effects of the residues of ivermectin in cattle dung using a sustained-release bolus on Aphodius constans (Duft.) (Coleoptera: Aphodiidae). Vet. Res., 32 , 421-427.

Eysker M., Ploeger H.W., 2000. Value of present diagnostic methods for gastrointestinal nematode infections in ruminants. Parasitology, 120, 109-119.

Eysker M., van Aarle D., Kooyman F.N.J., Nijzink A.M., Orsel K., Ploeger H.W., 2002. Exposure of dairy cows to nematode infections at the end of the grazing season in the Netherlands. Vet. Parasitol., 110, 93-100.

Floate K.D., 1998. Off-target effects of ivermectin on insects and on dung degradation in southern Alberta, Canada. Bull. Entomol. Res., $88,25-35$.

Forbes A.B., Huckle C.A., Gibb M.J., 2004. Impact of eprinomectin on grazing behaviour and performance in dairy cattle with sub-clinical gastro-intestinal nematode infections under continuous stocking management. Vet. Parasitol., $125,353-364$.

Forbes A.B., Vercruysse J., Charlier J., 2008. A survey of the exposure to Ostertagia ostertagi in dairy cow herds in Europe through the measurement of antibodies in milk samples from the bulk tank. Vet. Parasitol., 157, 100-107.

Fresnay E., 2004. Exemples de la mise en œuvre de la « cascade » dans le traitement des parasitoses chez les ruminants laitiers. Bull. GTV, Hors Série Parasitologie des Ruminants Laitiers, 140-144.

Gaba S., Cabaret J., Chylinski C., Sauvé C., Cortet J., Silvestre A., 2012. Can efficient management of sheep gastro-intestinal nematodes be based on random treatment? Vet. Parasitol., 190, 178-184.

Gayrard V., Alvinerie M., Toutain P.L., 1999 Comparison of pharmacokinetic profiles of doramectin and ivermectin «pour-on» formulations in cattle. Vet. Parasitol., 81, 47-55.

Geurden T., Hoste H., Jacquiet P., Traversa D. Sotiraki S., Frangipane di Regalbono A., Tzanidakis N., Kostopoulou D., Gaillac C., Privat S., Giangaspero A., Zanardello C., Noé L., Vanimisetti B., Bartram D., 2014. Anthelmintic resistance and multidrug resistance in sheep gastro-intestinal nematodes in France, Greece and Italy. Vet. Parasitol., 201, 59-66.

Geurden T., Chartier C., Fanke J., Frangipane di Regalbono A., Traversa D., von SamsonHimmelstjerna G., Demeler J., Vanimisetti H.B. Bartram D., Denwood M.J., 2015. Anthelmintic resistance to ivermectin and moxidectin in gastrointestinal nematodes of cattle in Europe. Int. J. Parasitol Drugs Drug Resist., 5, 163-171.

Gibb M.J., Huckle C.A. Forbes A.B., 2005 Effects of sequential treatments with eprinomectin on performance and grazing behaviour in dairy cattle under daily-paddock stocking management. Vet. Parasitol., 133, 79-90.

Grenfell B.T., Smith G., Anderson R.M., 1987. A mathematical model of the population biology of Ostertagia ostertagi in calves and yearlings. Parasitology, 95, 389-406.

Gross S.J., Ryan W.G., Ploeger H.W., 1999 Anthelminthic treatment of dairy cows and its effect on milk production. Vet. Rec., 144, 581587.

Grüner L., Raynaud J.P., 1980. Technique allégée de prélèvement d'herbe et de numération, pour juger de l'infestation des pâturages de bovins par les larves de nématodes parasites. Rev. Med. Vet., 131, 521-529.

Höglund J., Morrison D.A., Charlier J., Dimander S.O., 2009. Assessing the feasibility of targeted selective treatments for gastrointestinal nematodes in first-season grazing cattle based on mid-season daily weight gains. Vet. Parasitol., 164, 80-88.

Höglund J., Dahlströmb F., Sollenberga S. Hessle A., 2013. Weight gain-based targeted selective treatments (TST) of gastrointestinal nematodes in first-season grazing cattle. Vet. Parasitol., 196, 358-365.

Hoste H., Chartier C., 1993. Comparison of the effects on milk production of concurrent infection with Haemonchus contortus and Trichostrongylus colubriformis in high- and low-producing dairy goats. Am. J. Vet. Res., 54, 1886-1893.

Hoste H., Chartier C., Lefrileux Y., Goudeau C., Broqua C., Pors I., Bergeaud J.P., Dorchies P., 2002a. Targeted application of anthelmintics to control trichostrongylosis in dairy goats result from a two-year survey in farms. Vet. Parasitol., 110, 101-108.

Hoste H., Chartier C., Lefrileux Y., 2002b. Control of gastrointestinal parasitism with nematodes in dairy goats by treating the host category at risk. Vet. Res., 33, 531-545.

Hoste H., Cabaret J., Grosmond G., Guitard J.P., 2009. Alternatives aux traitements anthelminthiques en élevage biologique de ruminants.
In : Numéro spécial, Elevage bio. Perez J.M. (Ed). INRA Prod. Anim., 22, 245-254.

Hoste H., Sotiraki S., Landau S.Y., Jackson F., Beveridge I., 2010. Goat nematode interactions: think differently. Trends Parasitol., 26, 376-381.

Jackson F., Coop R.L., 2000. The development of anthelmintic resistance in sheep nematodes. Parasitology, 120, 95-107.

Jackson F., Varady M., Bartley D.J., 2012. Managing anthelmintic resistance in goats Can we learn lessons from sheep? Small Rum. Res., 103, 3-9.

Jacquiet P., Fidelle F., Lepetitcolin E., Privat S., Gaillac C., Bergeaud J.P., Hoste H., 2014a. Etat des lieux de la résistance aux anthelminthiques en France chez les ovins. Le Nouveau Prat. Vet. Elevage et santé n²9, 16-22.

Jacquiet P., Canellas A., Bonnefont M., Prevot F., Lienard E., Milhes M., Grisez C., 2014b. Les outils de dépistage des strongyloses gastrointestinales chez les ovins. Le Nouveau Prat. Vet. Elevage et santé n²9, 23-30.

Kaplan R.M., Vidyashankar A.N., 2012. An inconvenient truth: global worming and anthelmintic resistance. Vet. Parasitol., 186, 70-78.

Kenyon F., Jackson F., 2012. Targeted flock/herd and individual ruminant treatment approaches. Vet. Parasitol., 186, 10-17.

Kenyon F., Greer A.W., Coles G.C., Cringoli G., Papadopoulos E., Cabaret J., Berrag B., Varady M., van Wyk J.A., Thomas E., Vercruysse J., Jackson F., 2009. The role of targeted selective treatments in the development of refugia-based approaches to the control of gastrointestinal nematodes of small ruminants. Vet. Parasitol., 164, 3-11.

Kerboeuf D., Le Garff G., Mage C., 1981. Forecasting of bovine abomasal worm burden by means of serum pepsinogen measurements: study on suckling calves and heifers in first and second grazing season. Ann. Rech. Vet., 12, 201-213.

Kerboeuf D., Koch C., Le Dréan E., Lacourt A., 2002. Méthode simplifiée de mesure de la concentration en pepsinogène dans le sérum. Revue Méd. Vét., 153, 707-712.

Kulo A.E., Pors I., Chartier C., 2006. On-farm clinical indicators of parasitic nematode infection in French dairy goats: does-it work? 11 ${ }^{\text {th }}$ International Congress of Parasitology (ICOPA XI), 6-11 august 2006, Glasgow, Scotland.

Leathwick D.M., Besier R.B., 2014. The management of anthelmintic resistance in grazing ruminants in Australasia-strategies and experiences. Vet. Parasitol., 204, 44-54.

Lumaret J.P., Alvinerie M., Hempel H., Schallnass H.J., Claret D., Römbke J., 2007. New screening test to predict the potential impact of ivermectin-contaminated cattle dung on dung beetles. Vet. Res., 38, 15-24.

Lumaret J.P., Errouissi F., Floate K., Römbke J., Wardhaugh K., 2012. A Review on the Toxicity and Non-Target Effects of Macrocyclic Lactones in Terrestrial and Aquatic Environments. Curr. Pharm. Biotechnol., 13, 1004-1060.

Madsen M., Nielsen B.O., Holter P., Pedersen O.C., Jespersen J.B., Vagn Jensen K.M., Nansen P., Grønvold J., 1990. Treating cattle 
with ivermectins: effects on fauna and decomposition of dung pats. J. Appl. Ecol., 27, 1-15.

Mahieu M., Arquet R., Kandassamy T., Mandonnet N., Hoste H., 2007. Evaluation of targeted drenching using Famacha ${ }^{\circledR}$ method in Creole goat: reduction of anthelmintic use, and effects on kid production and pasture contamination. Vet. Parasitol., 146, 135-147.

Mahieu M., Ferré B., Madassamy M., Mandonnet N., 2014. Fifteen years later, anthelmintic resistances have dramatically spread over goat farms in Guadeloupe. Vet. Parasitol. $205,379-384$.

Mahieu M., Gauthier V., Arquet R., Calif B., Archimède H., Mandonnet N., 2015. Feasibility of a "leader-follower" grazing system instead of specialised paddocks with regard to integrated gastrointestinal control in small ruminant farming. Trop. Anim. Health Prod., 47, 773-778.

Mandonnet N., Aumont G., Fleury J., Arquet R., Varo H., Gruner L., Bouix J., Khang J.V., 2001. Assessment of genetic variability of resistance to gastrointestinal nematode parasites in Creole goats in the humid tropics. J. Anim. Sci. 79, 1706-1712.

Mandonnet N., Ducrocq V., Arquet R., Aumont G., 2003. Mortality of Creole kids during infection with gastrointestinal strongyles: a survival analysis. J. .Anim. Sci., 81, 2401-2408.

Manolaraki F., Boukary M., Pardo E., Pommaret A., Le Frileux Y., Thuault F., Cosnier K., Hoste H., 2014. Coproscopies de mélange et de broyat: des outils développés pour améliorer le suivi des infestations par les nématodes du tube digestif chez les chèvres. Renc. Rech. Rum., 339.

McKellar Q.A., 1997. Ecotoxicology and residues of anthelmintics compounds. Vet. Parasitol., 72, 413-435.

Mc Kenna P.B., 1985.Diagnosis of gastrointestinal nematode parasitism in goats. In: Proceedings of a course in Goat Husbandy and Medicine. Publication $n^{\circ} 106$, Veterinary Continuing Education, Massey University, Palmerston North, New-Zealand, 86-95.

Merlin A., Chauvin A., Madouasse A., Froger S., Bareille N., Chartier C., 2016a. Explaining variability in first grazing season heifer growth combining individually measured parasitological and clinical indicators with exposure to gastrointestinal nematode infection based on grazing management practices. Vet. Parasitol., 225, 6169.

Merlin A., Chauvin A., Lehebel A., Brisseau N., Froger S., Bareille N., Chartier C., 2016b. Evaluation de la faisabilité d'un traitement sélectif ciblé contre les strongles gastro-intestinaux chez les génisses laitières de première saison de pâturage basé sur la combinaison d'indicateurs liés à la conduite du pâturage et au GMQ individuel. Épidémiol. et Santé Anim., 2016, in press.

Nielsen M.K., 2009. Restrictions of anthelmintic usage : perspectives and potential consequences. Parasit. Vectors, 25 Suppl 2:S7. doi: 10.1186/ 1756-3305-2-S2-S7.

O'Shaughnessy J., Earley B., Mee J.F., Doherty M.L., Crosson P., Barrett D., de Waal, T., 2015. Controlling nematodes in dairy calves using targeted selective treatments. Vet. Parasitol., 209, 221-228.
Paraud C., Pors I., Kulo A., Chartier C., 2007. Application d'une stratégie de traitement cible dans le cadre de la résistance aux anthelminthiques chez les caprins. In : Proc. Journ. Nat. GTV, Nantes, France, 623-632.

Paraud C., Kulo A., Pors I., Chartier C., 2009. Resistance of goat nematodes to multiple anthelminthics on a farm in France. Vet. Rec. $164,563-564$

Paraud C., Pors I., Marcotty T., Devos J., 2014 Un premier cas de résistance aux lactones macrocycliques chez les nématodes gastrointestinaux confirmé en élevage ovin en France. Renc. Rech. Rum., 21, 325-328.

Ploeger H.W., Schoenmaker G.J.W., Kloosterman A., Borgsteede F.H.M., 1989. Effect of anthelminthic treatment of dairy cattle on milk production related to some parameters estimating nematode infection. Vet. Parasitol., 34, 239-253.

Ploeger H.W., Kloosterman A., Bargeman G., Wuijckhuise L., Van Der Brink R., 1990. Milk yield increase after anthelminthic treatment of dairy cattle related to some parameters estimating helminth infection. Vet. Parasitol., 35, 103 116

Pritchard R., Ménez C., Lespine A., 2012 Moxidectin and the avermectins : consanguinity but not identity. Int. J. Parasitol. Drugs Drug Resist., 2, 134-153.

Putman R.J., 1983. Carrion and dung: the decomposition of animal wastes. Inst. Biol. Stud. Biol., 156, 62 p.

Ravinet N., Bareille N., Lehebel A., Ponnau A. Chartier C., Chauvin A., 2014. Change in milk production after treatment against gastrointestinal nematodes according to grazing history, parasitological and production-based indicators in adult dairy cows. Vet. Parasitol., 201, 95 109.

Ravinet N., Merlin A., Chartier C., Bareille N., Chauvin A., 2015a. Strongyloses digestives chez les bovins : variabilités des conséquences cliniques et zootechniques. In : Proc. Journ. Nat. GTV, Nantes, France, 91-102.

Ravinet N., Lehebel A., Lopez C., Madouasse A., Brisseau N., Ponnau A., Chartier C., Bareille N., Chauvin A., 2015b. Stratégie de traitemen anthelminthique ciblé-sélectif : exemple chez la vache laitière. Bull. GTV, 80, 31-40.

Ravinet N., Chartier C., Bareille N., Lehebel A., Ponnau A., Brisseau N., Chauvin A., 2016. Unexpected decrease in milk production after fenbendazole treatment of dairy Cows during early grazing season. Plos One, 11, doi: 10.1371/ journal.pone.0147835

Rose H., Rinaldi L., Bosco A., Mavrot F., de Waal T., Skuce P., Charlier J., Torgerson P.R. Hertzberg H., Hendrickx G., Vercruysse J. Morgan E.R., 2015. Widespread anthelmintic resistance in European farmed ruminants: a systematic review. Vet. Rec., doi:10.1136/ vr.102982

Sanchez J., Dohoo I., Nødtvedt A., Keefe G., Markham F., Leslie K., DesCôteaux L. Campbell J., 2002. A longitudinal study of gastrointestinal parasites in Canadian dairy farms. The value of an indirect Ostertagia ostertagi ELISA as a monitoring tool. Vet. Parasitol. 107, 209-226.
Sanchez J., Dohoo I., Carrier J., DesCôteaux L., 2004. A meta-analysis of the milk-production response after anthelminthic treatment in naturally infected adult dairy cows. Prev. Vet. Med., 63, 237-256.

Scheuerle M., Mahling M., Muntwyler J., Pfister K., 2010. The accuracy of the FAMACHA(C-method in detecting anemia and haemonchosis in goat flocks in Switzerland under field conditions. Vet. Parasitol., 170, 71-77.

Shaw D.J., Vercruysse J., Claerebout E., Agneessens J., Dorny P., 1997. Gastrointestinal nematode infections of first-season grazing calves in Belgium: general patterns and the effect of chemoprophylaxis. Vet. Parasitol., 69, 103-116.

Shaw D.J., Vercruysse J., Claerebout E., Dorny P., 1998a. Gastrointestinal nematode infections of first-grazing season calves in Western Europe: general patterns and the effect of chemoprophylaxis. Vet. Parasitol., 75, 115-131.

Shaw D.J., Vercruysse J., Claerebout E., Dorny P., 1998b. Gastrointestinal nematode infections of first-grazing season calves in Western Europe: Associations between parasitological, physiological and physical factors. Vet. Parasitol., 75, 133-151.

Sommer C., Steffansen B., 1993. Changes with time after treatment in the concentrations of ivermectin in fresh cow dung and in cow pats aged in the field.Vet. Parasitol., 48, 67-73.

Stafford K., Coles G.C., 1999. Nematode control practices and anthelminthic resistance in dairy calves in the south west of England. Vet. Rec., 144, 659-661.

Stromberg B.E., 1997. Environmental factors influencing transmission. Vet. Parasitol., 72, 247-264.

Sutherland I.A., Leathwick D.M., 2011. Anthelmintic resistance in nematode parasites of cattle: a global issue? Trends Parasitol., 27, 176-180.

Tharaldsen J., Helle O., 1989. The effect of periparturient treatment with fenbendazole on the milk production of cows. Acta Vet. Scand., $30,247-252$.

van Wyk J.A., 2001. Refugia - overlooked as perhaps the most important factor concerning the development of anthelmintic resistance. Onderstepoort J. Vet. Res., 68, 55-67.

van Wyk J., Hoste H., Kaplan R., Besier R.B., 2006. Targeted selective treatment for worm management - How do we sell rational programs to farmers? Vet. Parasitol., 139, 336346.

Vanderstichel R., Dohoo I., Sanchez J., Sithole F., Keefe G., Stryhn H., 2013. Predicting the effect of anthelmintic treatment on milk production of dairy cattle in Canada using an Ostertagia ostertagi ELISA from individual milk samples. Prev. Vet. Med., 111, 63-75.

Vercruysse J., Hilderson H., Claerebout E., 1994. Effect of chemoprophylaxis on immunity to gastrointestinal nematodes in cattle. Parasitology Today, 4, 129-132.

Vercruysse J., Claerebout E., 1997. Immunity development against Ostertagia ostertagi and other gastrointestinal nematodes in cattle. Vet. Parasitol., 72, 309-326. 
Verschave SH, Vercruysse J, Forbes A, Opsomer G, Hostens M, Duchateau L, Charlier J., 2014. Noninvasive indicators associated with the milk yield response after anthelmintic treatment at calving in dairy cows. BMC Vet. Res., 10:264 - doi:10.1186/s12917-014-0264-x.

\section{Résumé}

Chez les ruminants élevés au pâturage, les strongles gastro-intestinaux (SGI) peuvent induire des pertes de production, voire des atteintes cliniques chez les animaux non immuns. Le contrôle de l'infestation repose essentiellement sur les traitements anthelminthiques $(\mathrm{AH})$ administrés fréquemment sans évaluation préalable de la réalité du risque parasitaire. Cette synthèse vise tout d'abord à exposer les principaux risques associés à ce recours insuffisamment raisonné aux $\mathrm{AH}$ : apparition de populations de parasites résistants aux $\mathrm{AH}$, écotoxicité pour la microfaune prairiale dégradant les fèces, et installation retardée de l'immunité anti-strongles (notamment chez les bovins). Les stratégies de traitement ciblé-sélectif devraient permettre de maîtriser ces risques tout en prévenant les atteintes cliniques et sécurisant les performances des animaux. Il s'agit de rationaliser les traitements AH en ciblant les troupeaux ou les lots et les périodes à risque, et en sélectionnant les individus les plus parasités ou « souffrant » le plus du parasitisme. Cette synthèse vise donc ensuite à décrire les outils et les indicateurs étudiés pour mettre en ouvre de telles stratégies chez les ruminants. Il s'agit d'indicateurs zootechniques (gain moyen quotidien, parité, niveau de production...), cliniques (FAMACHA@), index de diarrhée...), parasitologiques (coproscopie, niveau d'anticorps anti-SGI...), ou d'indicateurs de conduite (modalité de pâturage), et aussi d'outils informatiques (modélisation du recyclage parasitaire). Leur fiabilité et leur opérationnalité sont variables. La mise en place de telles stratégies nécessitera de dépasser les freins à l'acceptation de ces nouvelles pratiques tant au niveau des éleveurs que des vétérinaires prescripteurs. Cet usage raisonné des AH assurerait une meilleure durabilité du contrôle de l'infestation par les SGI.

\section{Abstract}

Issues and tools for an evidence-based use of treatments against gastrointestinal nematodes in cattle and small ruminants

In grazing ruminants, gastrointestinal nematode (GIN) infection can induce production losses, even clinical disease in non-immunized animals. Control measures essentially rely on anthelmintic $(\mathrm{AH})$ treatments, applied frequently and without prior assessment of the effective parasitological risk. This review aims firstly at putting forward the main risks related to this non rational use of AH: emergence of drug-resistant parasitic populations, ecotoxicity on the microfauna involved in feces degradation on pastures, and delayed development of immunity against GIN (particularly in cattle). Targeted-selective treatment strategies should put such risks under control, while preventing clinical disease and securing productions. AH treatment would be rationalized by targeting herds/flocks, groups and periods with a parasitological risk, and by selecting the less resistant/resilient animals. Thus, this review aims secondly at describing the tools and indicators studied to implement such strategies: production-based indicators (daily weight gain, parity, level of production...), clinical indicators (FAMACHA $\odot$, diarrhea index...), parasitological indicators (coproscopy, anti-GIN antibody level...), or indicators based on grazing management, and software tools (simulation model of the pasture infectivity level). Their reliability and operationality are variable. The implementation of these innovative strategies is a paradigm shift with respect to current practices, and requires the lowering of acceptance barriers. Such a rational use of AH would ensure the sustainability of control measures against GIN infection in ruminants.

RAVINET N., CHARTIER C., HOSTE H., MAHIEU M., DUVAUCHELLE-WACHE A., MERLIN A., BAREILLE N., JACQUIET P., CHAUVIN A., 2017. Enjeux et outils du traitement raisonné contre les strongles gastro-intestinaux chez les bovins et les petits ruminants. INRA Prod. Anim., 30, 57-76. 
